\title{
Recommandations
}

\section{Prise en charge des foyers infectieux bucco-dentaires}

\author{
Société Française de Chirurgie Orale

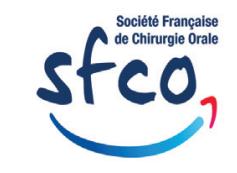

Composition des groupes de travail

\section{Groupe de pilotage :}

- Jean-Hugues CATHERINE

- Benoit LEFEVRE

- Laurent NAWROCKI

- Jacques-Henri TORRES

\section{Groupe de cotation G1 :}

- René-Jean BENSADOUN

- Patrick BLANCHARD

- Jean-Hugues CATHERINE

- Eric DENES

- Vincent ESNAULT

- José GOMES

- Raoul HERBRECHT

- Bernard IUNG

- Nicolas MAILHAC

- Jean-Pierre MARIOTTINI

- Laurent NAWROCKI

- Jean-Michel RISTORI

- Henri tenenbaum

- Jacques-Henri TORRES

- Loïc VAILLANT

(Radiothérapie, Poitiers)

(Stomatologie, Maisons-Laffitte)

(Médecine Bucco-Dentaire, Marseille) Rapporteur

(Infectiologie, Limoges)

(Néphrologie, Nice)

(Médecine Générale, Mornac)

(Hématologie, Strasbourg)

(Cardiologie, Paris)

(Médecine Bucco-Dentaire, Autun)

(Médecine Bucco-Dentaire, Nice)

(Médecine Bucco-Dentaire, Lille) Coordinateur

(Rhumatologie, Clermont-Ferrand)

(Médecine Bucco-Dentaire, Strasbourg)

(Stomatologie, Montpellier) Président

(Dermatologie, Tours)

\section{Groupe de cotation G2 :}

- Patrick BLANCHARD

- Sylvie BOISRAME-GASTRIN

- Jean-Hugues CATHERINE

- Anne-Gaëlle CHAUX-BODARD

- Benoit LOTH

- Eric MALAdIERE

- Nicolas MAILHAC

- Jean-Pierre MARIOTTINI

- Laurent NAWROCKI

- Henri TENENBAUM

- Jacques-Henri TORRES

(Stomatologie, Maisons Laffitte)

(Médecine Bucco-Dentaire, Brest)

(Médecine Bucco-Dentaire, Marseille) Rapporteur

(Médecine Bucco-Dentaire, Lyon)

(Médecine Bucco-Dentaire, Eckbolscheim)

(Stomatologie, Perpignan)

(Médecine Bucco-Dentaire, Autun)

(Médecine Bucco-Dentaire, Nice)

(Médecine Bucco-Dentaire, Lille) Coordinateur

(Médecine Bucco-Dentaire, Strasbourg)

(Stomatologie, Montpellier) Président 


\section{Groupe de lecture :}

- Pierrick ARCHAMBAULT

- Yves COTTIN

- Xavier DUFOUR

- Thierry DUMOUSSEAU

- Xavier DUVAL

- Joël FERRI

- Camille FRANCES

- Jean-Christophe FRICAIN

- Pierre GANGLOFF

- Laurent GUYOT

- Gilbert HABIB

- Rozenn LE BERRE

- Sandrine MALOCHET-GUINAMAND

- Olivier PRADIER

- Vincent RONCO

- Jean-Philippe SANNAJUST

- Christine SELTON-SUTY

- Juliette THARIAT

- Gérald VALETTE

- Thierry VANNUFFEL

- Vincent VIDAL

- Catherine VIGNAL-CLERMONT
(Médecine Générale, Nueil-les-Aubiers)

(Cardiologie, Dijon)

(Oto-Rhino-Laryngologie, Poitiers)

(Chirurgie Maxillo-Faciale et Stomatologie, Seclin)

(Infectiologie, Paris)

(Chirurgie Maxillo-Faciale et Stomatologie, Lille)

(Dermatologie, Paris)

(Médecine Bucco-Dentaire, Bordeaux)

(Médecine Bucco-Dentaire, Metz)

(Chirurgie Maxillo-Faciale, Marseille)

(Cardiologie, Marseille)

(Infectiologie, Brest)

(Rhumatologie, Clermont-Ferrand)

(Oncologie Radiothérapie, Brest)

(Médecine Bucco-Dentaire, Paris)

(Chirurgie Maxillo-Faciale/Chirurgie Générale, Draguignan)

(Cardiologie, Nancy)

(Oncologie Radiothérapie, Nice)

(Oto-Rhino-Laryngologie/Oncologie, Brest)

(Médecine Bucco-Dentaire, Binche)

(Chirurgie Cardio-Thoracique et Vasculaire, Nîmes)

(Ophtalmologie, Paris) 


\section{Synthèse des recommandations}

Le risque infectieux lié aux actes pratiqués en cabinet dentaire doit être relativisé. Il y a en effet beaucoup plus de bactériémies induites par les actes de la vie quotidienne (brossage dentaire, mastication) que par des soins dentaires. Le spécialiste de la cavité buccale reçoit fréquemment des patients adressés par des praticiens de différentes spécialités pour rechercher et éradiquer les foyers infectieux bucco-dentaires avant transplantation d'organe, instauration d'un traitement immunosuppresseur, chimiothérapie, radiothérapie, chirurgie valvulaire.

Le bilan bucco-dentaire doit impérativement comprendre un examen clinique complet (interrogatoire, sondage parodontal, tests de vitalité, percussion, palpation des chaînes ganglionnaires...) ainsi qu'un examen radiographique panoramique, qui doit être complété en cas de doute par d'autres examens (clichés rétro-alvéolaires, cone beam, scanner).

Dans tous les cas, les gestes chirurgicaux destinés à assainir la cavité buccale doivent être entrepris au plus tôt, de façon à ce que la cicatrisation muqueuse soit acquise avant l'apparition du risque infectieux supplémentaire.

Chez les patients présentant un risque infectieux particulier, la cicatrisation muqueuse après avulsion dentaire nécessite une durée minimale d'une semaine. Elle doit être évaluée par un contrôle clinique.

Certaines situations sont susceptibles de majorer le risque infectieux tant qu'une plaie n'est pas refermée. C'est le cas des patients présentant un risque d'ostéoradionécrose, des diabétiques non équilibrés (hémoglobine glyquée $>7 \%$ ), ou des sujets chez qui le taux de polynucléaires neutrophiles est inférieur à $500 / \mathrm{mm}^{3}$ de sang. Dans ces situations, un traitement antibiotique doit bien entendu être institué avant le geste invasif, mais aussi poursuivi jusqu'à la cicatrisation de la muqueuse.

En cas d'infection bucco-dentaire survenant chez un patient fragile par ailleurs (radiothérapie, chimiothérapie, risque infectieux valvulaire...), le contexte médical général ne doit jamais constituer un prétexte pour retarder la prise en charge de l'urgence infectieuse (en particulier, un abcès doit être drainé).

Une corticothérapie par voie générale ne justifie pas une prise en charge particulière du fait de sa seule présence si elle a une posologie inférieure à $10 \mathrm{mg} / \mathrm{j}$ équivalent prednisone ou si elle a une durée de moins de 8 jours pour une posologie inférieure ou égale à $1 \mathrm{mg} / \mathrm{Kg} / \mathrm{j}$ équivalent prednisone.

De la même façon, il n'y a pas lieu de prendre des mesures particulières si la dose d'irradiation reçue par le maxillaire et/ou la mandibule est inférieure à $30 \mathrm{~Gy}$.

Lors de l'examen clinique chez les patients à haut risque d'endocardite infectieuse, le sondage parodontal doit être réalisé sous antibioprophylaxie.

Chez les patients traités ou ayant été traités par bisphosphonates en dehors du cadre d'une pathologie maligne, les avulsions peuvent faire l'objet d'une prise en charge en cabinet d'omnipratique dentaire, dans le respect des règles de prise en charge. De même, les patients diabétiques peuvent faire l'objet d'une prise en charge en cabinet d'omnipratique dentaire, après vérification de l'équilibre du diabète. En revanche, chez les patients traités ou ayant été traités par bisphosphonates dans le cadre d'une pathologie maligne, ainsi que chez les patients présentant un risque d'ostéoradionécrose, il est recommandé de pratiquer les avulsions dans un plateau technique chirurgical adapté à la situation et apportant les garanties de qualité et de sécurité (par exemple en milieu hospitalier).

En cas de risque infectieux particulier (autre que dentaire), les différentes possibilités de traitements des foyers infectieux bucco-dentaires doivent être expliquées au patient, et son consentement éclairé doit être recueilli.

Le choix de la thérapeutique retenue doit intégrer en particulier :

- le pronostic vital du patient lié à l'affection générale ;

- le risque lié à l'abstention thérapeutique ;

- la morbidité inhérente à chaque solution thérapeutique ;

- le bénéfice attendu de chaque proposition thérapeutique sur le confort de vie ;

- l'observance prévisible aux manœuvres d'hygiène et aux visites de contrôle.

Afin d'éclairer le praticien dans son choix thérapeutique et en l'absence de données dans la littérature, les experts ont exprimé dans un diagramme leur estimation du risque infectieux pour les situations les plus fréquentes. Le risque le plus important correspond aux cellulites péri-maxillaires, aux péricoronarites aiguës et aux parodontites agressives. 


\section{Principaux messages}

\section{Introduction}

Le spécialiste de la cavité buccale reçoit fréquemment des patients adressés par des praticiens de différentes spécialités pour une recherche de foyers infectieux bucco-dentaires (FIBD).

La recherche de FIBD est demandée par le correspondant dans plusieurs circonstances :

- en raison d'un état général particulier, pour prévenir l'apparition d'une infection secondaire ou pour stabiliser une pathologie générale ;

- $\quad$ pour rechercher le point de départ d'une infection secondaire ;

- avant d'instaurer une thérapeutique médicale (chimiothérapie, thérapies immunosuppressives) ou physique (radiothérapie) susceptible de favoriser ou d'aggraver un processus infectieux ;

- pour préparer le patient à une intervention chirurgicale.

Seuls sont traités dans ces recommandations les foyers infectieux d'origine bactérienne. En sont par ailleurs exclues les pathologies infectieuses muqueuses, osseuses d'origine non dentaire et salivaires.

On peut définir :

- des FIBD (actifs ou latents) : présence effective de foyers bactériens, qu'il s'agisse d'une infection avérée ou qu'il n'y ait pas de répercussion clinique au moment de l'observation ;

- des situations à risque infectieux potentiel (SRIP) : susceptibles de devenir des foyers infectieux dans le futur du fait des conditions réunies à l'échelon local.

\section{Bactériémie, virulence infectieuse (voir diagramme)}

Il y a beaucoup plus de bactériémies induites par les actes de la vie quotidienne (brossage dentaire, mastication) que par des soins dentaires (NP 2).

Une dent vitale atteinte d'une carie dont le traitement ne comporte pas de risque d'effraction pulpaire ne représente pas un foyer infectieux susceptible de dissémination (APF).

Par comparaison avec le traitement endodontique d'une dent vitale,

- le traitement endodontique d'une dent nécrosée,

- une reprise de traitement endodontique représentent un risque majoré d'entraîner une bactériémie (APF).

Une dent nécrosée représente un FIBD ou une SRIP (APF).

Les dents en désinclusion des foyers infectieux actifs ou latents (APF).

\section{Population générale}

Abréviations :

FIDB : foyer infectieux bucco-dentaire SRIP : situation à risque infectieux potentiel Niveau de preuve 1 : preuve scientifique établie Niveau de preuve $\mathbf{2}$ : présomption scientifique Niveau de preuve 3 : faible niveau de preuve scientifique APF : accord professionnel fort

CR : accord professionnel avec consensus relatif

Pour l'ensemble de la population, indépendamment de toute notion de pathologie :

1) Il est recommandé d'éliminer les FIBD (APF) ainsi que les SRIP (CR).

2) La découverte d'un FIBD doit faire l'objet d'une information délivrée au patient, précisant les conséquences éventuelles de cette infection (APF).

3) La découverte d'une SRIP doit faire l'objet d'une information délivrée au patient (APF), contenant l'évaluation du risque de développement d'une infection (CR) et précisant les conséquences éventuelles de cette infection (APF).

\section{Conduite de la recherche de foyers infectieux}

4) Un bilan bucco-dentaire doit impérativement comprendre un examen clinique (interrogatoire, sondage parodontal, tests de vitalité, percussion, palpation des chaînes ganglionnaires...) (APF). Voir aussi la recommandation $n^{\circ} 28$. 
5) Le bilan initial doit impérativement comprendre un examen radiographique panoramique (APF).

6) En cas de doute à la lecture de l'examen panoramique, l'examen radiographique doit être complété par d'autres examens : clichés rétro-alvéolaires, tomographie volumique à faisceau conique (cone beam), tomodensitométrie (scanner) (APF).

\section{Attitude générale chez les patients présentant un risque infectieux particulier (autre que dentaire)}

7) Un contact préalable avec le médecin responsable du suivi du patient est recommandé pour évaluer le risque médical (APF).

8) En cas de survenue d'une infection bucco-dentaire, le contexte médical général (radiothérapie, chimiothérapie, chirurgie cardiaque...) ne doit pas retarder la prise en charge chirurgicale et/ou médicale de l'urgence infectieuse. En particulier, un abcès doit être drainé (APF).

\section{Décision thérapeutique chez les patients présentant un risque infectieux particulier}

9) Dans les cas de FIBD, comme dans les SRIP, le choix de la thérapeutique bucco-dentaire doit intégrer des notions multiples, en particulier (APF) :

- le pronostic vital du patient lié à l'affection générale ;

- le risque lié à l'abstention thérapeutique, en fonction notamment de la virulence présumée du foyer infectieux ;

- la morbidité inhérente à chaque solution thérapeutique ;

- le bénéfice attendu de chaque proposition thérapeutique pour le confort de vie du patient ;

- l'observance prévisible du patient aux manœuvres d'hygiène et aux visites de contrôle.

10) Dans tous les cas, les différents traitements doivent être expliqués au patient et son consentement éclairé doit être recueilli (APF).

\section{Contrôle de la cicatrisation chez les patients présentant un risque infectieux particulier}

11) La cicatrisation muqueuse après avulsion dentaire nécessite une durée minimale d'une semaine. Elle doit être évaluée par un contrôle clinique (APF).

\section{Radiothérapie cervico-faciale}

\section{Avant irradiation}

12) Avant toute radiothérapie cervico-faciale (APF) :

- un bilan bucco-dentaire doit être entrepris au plus tôt ;

- il est impératif d'éliminer les FIBD situés dans les faisceaux (champs) d'irradiation.

13) Les gestes chirurgicaux destinés à assainir la cavité buccale doivent être entrepris au plus tôt de façon à ce que la cicatrisation muqueuse soit acquise avant le début de la radiothérapie (APF).

Après irradiation (et quel que soit le délai)

14) Il n'y a pas lieu de prendre des mesures particulières si la dose d'irradiation reçue par le maxillaire et/ou la mandibule est inférieure à $30 \mathrm{~Gy}(\mathrm{CR})$.

15) Afin de diminuer le risque d'ostéoradionécrose, les avulsions dentaires éventuellement nécessaires doivent être effectuées :

- après information sur la dose reçue et les faisceaux (champs) d'irradiation (APF) ;

- dans un plateau technique chirurgical adapté à la situation et apportant les garanties de qualité et de sécurité (APF).

16) Devant un risque d'ostéoradionécrose, un traitement antibiotique doit être institué en cas de geste invasif (avulsion dentaire, curetage...) (APF). Il convient de débuter cette prescription au moins $1 \mathrm{~h}$ avant le geste (APF) (pas de consensus pour un délai plus long) et de la poursuivre jusqu'à la cicatrisation de la muqueuse (APF).

17) Après irradiation supérieure à $30 \mathrm{~Gy}$, un suivi bucco-dentaire est recommandé avec une fréquence de 4 à 6 mois (CR). 


\section{Corticothérapie}

18) Une corticothérapie par voie générale ne justifie pas une prise en charge particulière du fait de sa seule présence :

- si elle a une posologie inférieure à $10 \mathrm{mg} / \mathrm{j}$ équivalent prednisone ou

- $\quad$ si elle a une durée de moins de 8 jours pour une posologie inférieure ou égale à $1 \mathrm{mg} / \mathrm{Kg} / \mathrm{j}$ équivalent prednisone (APF).

\section{Transplantation, Immunodépression}

19) Avant une transplantation ou l'instauration d'un traitement immunosuppresseur, et dans la mesure où l'urgence à instaurer le traitement l'y autorise :

- un bilan bucco-dentaire doit être effectué au plus tôt (APF) ;

- il est impératif d'éliminer les FIBD (APF) ;

- les gestes chirurgicaux destinés à assainir la cavité buccale doivent être entrepris au plus tôt, de façon à ce que la cicatrisation muqueuse soit acquise avant la transplantation ou l'instauration d'un traitement immunosuppresseur (APF).

20) Chez les patients transplantés, un suivi bucco-dentaire est recommandé avec une fréquence de 4 à 6 mois (CR).

21) Lors de la découverte d'une séropositivité VIH, il est souhaitable de réaliser un bilan bucco-dentaire à la recherche de FIBD (APF).

22) Chez les patients atteints d'un SIDA, un suivi bucco-dentaire est recommandé avec une fréquence de 4 à 6 mois (CR).

\section{Chimiothérapie}

Avant la chimiothérapie

23) Dans la mesure où l'urgence à instaurer le traitement l'y autorise :

- un bilan bucco-dentaire doit être entrepris au plus tôt (APF) ;

- avant une chimiothérapie non aplasiante, il est particulièrement ${ }^{*}{ }^{*}$ recommandé d'éliminer les FIBD (APF) ;

- avant une chimiothérapie aplasiante, il est impératif d'éliminer les FIBD (APF) ;

24) Les gestes chirurgicaux destinés à assainir la cavité buccale doivent être entrepris au plus tôt, de façon à ce que la cicatrisation muqueuse soit acquise avant le début de la chimiothérapie (APF).

Pendant la chimiothérapie

25) Les gestes thérapeutiques invasifs (avulsion dentaire...) doivent être pratiqués :

- en connaissance du bilan biologique (hémogramme, hémostase) (APF) ;

- seulement s'ils sont urgents (CR) ;

- sous antibioprophylaxie poursuivie jusqu'à la cicatrisation muqueuse de la plaie si le taux de polynucléaires neutrophiles (PN) est inférieur à $500 / \mathrm{mm}^{3}$ de sang (APF). Il n'y a pas de consensus pour une antibioprophylaxie pour un taux de PN supérieur à $500 / \mathrm{mm}^{3}$ (PC).

26) En dehors des cas d'urgence, les gestes chirurgicaux peuvent être pratiqués dans une phase de normalité des polynucléaires neutrophiles (APF).

\section{Patient à risque d'endocardite infectieuse}

27) Un bilan bucco-dentaire doit être entrepris au plus tôt avant la chirurgie valvulaire (APF).

28) Lors de l'examen clinique chez les patients à haut risque d'endocardite infectieuse, le sondage parodontal doit être réalisé sous antibioprophylaxie (APF).

\begin{tabular}{|l|}
\hline \multicolumn{1}{|c|}{ Situations à haut risque d'endocardite infectieuse } \\
\hline Prothèse valvulaire cardiaque \\
\hline Antécédent d'endocardite \\
\hline Cardiopathie congénitale : \\
- cyanogène non réparée, y compris shunts et conduits palliatifs \\
- complètement réparée avec matériel prothétique, placé par cathétérisme ou \\
chirurgicalement, pendant les 6 mois suivant la procédure \\
- réparée avec défauts résiduels sur le site ou adjacent au site du patch \\
prothétique \\
\hline
\end{tabular}

${ }^{*}$ ) Par rapport au patient sain, chez qui il est recommandé d'éliminer les foyers infectieux 
29) Il est particulièrement $\left({ }^{*}\right)$ recommandé d'éliminer les FIBD chez les sujets à risque modéré d'endocardite infectieuse (APF).

30) Il est impératif d'éliminer les FIBD chez les sujets à haut risque d'endocardite infectieuse (APF).

31) Les gestes chirurgicaux destinés à assainir la cavité buccale doivent être entrepris au plus tôt de façon à ce que la cicatrisation muqueuse soit acquise avant la chirurgie valvulaire (APF).

32) Chez les patients à haut risque d'endocardite infectieuse, un suivi bucco-dentaire est recommandé avec une fréquence de 4 à 6 mois (CR).

33) Chez les sujets à risque modéré d'endocardite infectieuse, la fréquence de suivi peut être identique à celle recommandée pour la population générale (annuelle) (CR).

\section{Prothèses articulaires}

34) Avant la mise en place d'une prothèse articulaire :

- il est recommandé d'effectuer un bilan bucco-dentaire (APF)

- il est impératif d'éliminer les FIBD (CR).

35) Les gestes chirurgicaux destinés à assainir la cavité buccale doivent être entrepris au plus tôt de façon à ce que la cicatrisation muqueuse soit acquise avant la pose de la prothèse articulaire (APF).

36) Chez le sujet porteur de prothèse articulaire, la fréquence de suivi bucco-dentaire peut être identique à celle recommandée pour la population générale (annuelle) (CR).

\section{Thérapies biologiques ciblées à visée immunosuppressive (anticorps monoclonaux : anti-TNF alpha,} anti-lymphocytaires...)

37) Il est impératif d'éliminer les FIBD avant l'instauration d'une thérapie biologique ciblée à visée immunosuppressive (CR).

38) Si des soins invasifs (avulsions dentaires, pose d'implants...) sont envisagés pendant le traitement, une attention particulière doit être portée à la survenue potentielle d'infections post-opératoires : vigilance, information du patient. La décision thérapeutique sera prise au cas par cas en pesant le rapport bénéfice/risque avec le médecin prescripteur, qui proposera éventuellement une mise en condition du patient (pouvant comprendre la suspension du traitement) (APF).

\section{Traitements bisphosphonates}

39) À l'instauration d'un traitement par bisphosphonates (quelle qu'en soit l'indication), il est recommandé de pratiquer un bilan bucco-dentaire (CR).

40) Il est particulièrement ${ }^{*}$ ) recommandé d'éliminer les FIBD avant traitement par bisphosphonates (quelle qu'en soit l'indication) (APF).

41) Chez les patients traités ou ayant été traités par bisphosphonates dans le cadre d'une pathologie maligne :

- il est recommandé de pratiquer les avulsions dans un plateau technique chirurgical adapté à la situation et apportant les garanties de qualité et de sécurité (APF) ;

- un suivi bucco-dentaire est recommandé avec une fréquence de 4 à 6 mois (CR).

42) Chez les patients traités ou ayant été traités par bisphosphonates en dehors du cadre d'une pathologie maligne :

- les avulsions peuvent faire l'objet d'une prise en charge en cabinet d'omnipratique dentaire, dans le respect des règles de prise en charge (CR) ;

- la fréquence de suivi dentaire peut être identique à celle recommandée pour la population générale (CR).

\section{Pathologies respiratoires chroniques}

43) Il est particulièrement ${ }^{*}$ ) recommandé d'éliminer les FIBD chez les patients présentant des pathologies respiratoires chroniques (bronchopneumopathie obstructive chronique, asthme...) (APF).

$\left(^{*}\right)$ Par rapport au patient sain, chez qui il est recommandé d'éliminer les foyers infectieux 


\section{Patients diabétiques}

L'éradication des FIBD améliore le contrôle de la glycémie chez les patients diabétiques (NP 1).

44) Dès le diagnostic d'un diabète, il est recommandé de pratiquer un bilan bucco-dentaire (APF).

45) Les patients diabétiques peuvent faire l'objet d'une prise en charge en cabinet d'omnipratique dentaire, après vérification de l'équilibre du diabète (APF).

46) Il est particulièrement ${ }^{(*)}$ recommandé d'éliminer les FIBD chez les patients diabétiques équilibrés (APF).

47) Chez les patients diabétiques non équilibrés (hémoglobine glyquée $>7 \%$ ) :

- il est impératif d'éliminer les FIBD (CR) ;

- en cas de geste invasif, il faut commencer le traitement antibiotique prophylactique dans l'heure qui précède le geste et le poursuivre jusqu'à cicatrisation muqueuse de la plaie (CR) ;

- un suivi bucco-dentaire est recommandé avec une fréquence de 4 à 6 mois (CR).

\section{Grossesse}

Le traitement parodontal est réalisable pendant la grossesse (de préférence au $2^{\mathrm{e}}$ trimestre) mais ne permettrait pas une diminution des naissances prématurées ou des insuffisances pondérales du nouveau né (NP 2).

48) Chez les femmes enceintes ou ayant un projet de grossesse, il est recommandé de pratiquer un bilan bucco-dentaire (APF).

49) Il est particulièrement ${ }^{*}{ }^{*}$ recommandé d'éliminer les FIBD chez une femme ayant un projet de grossesse (APF).

\section{Situations sans niveau de preuve}

Il n'y a pas de preuve pour affirmer qu'un FIBD peut être à l'origine d'une tendinite.

De même, un consensus se dégage pour considérer qu'il n'y a pas lieu de rechercher une origine bucco-dentaire devant un cas de pelade ou d'uvéite.

$\left({ }^{*}\right)$ Par rapport au patient sain, chez qui il est recommandé d'éliminer les foyers infectieux 
Virulence présumée dans diverses situations cliniques

En l'absence de données de la littérature, les experts du groupe de pilotage et de cotation proposent ce diagramme destiné à éclairer le praticien dans son choix thérapeutique

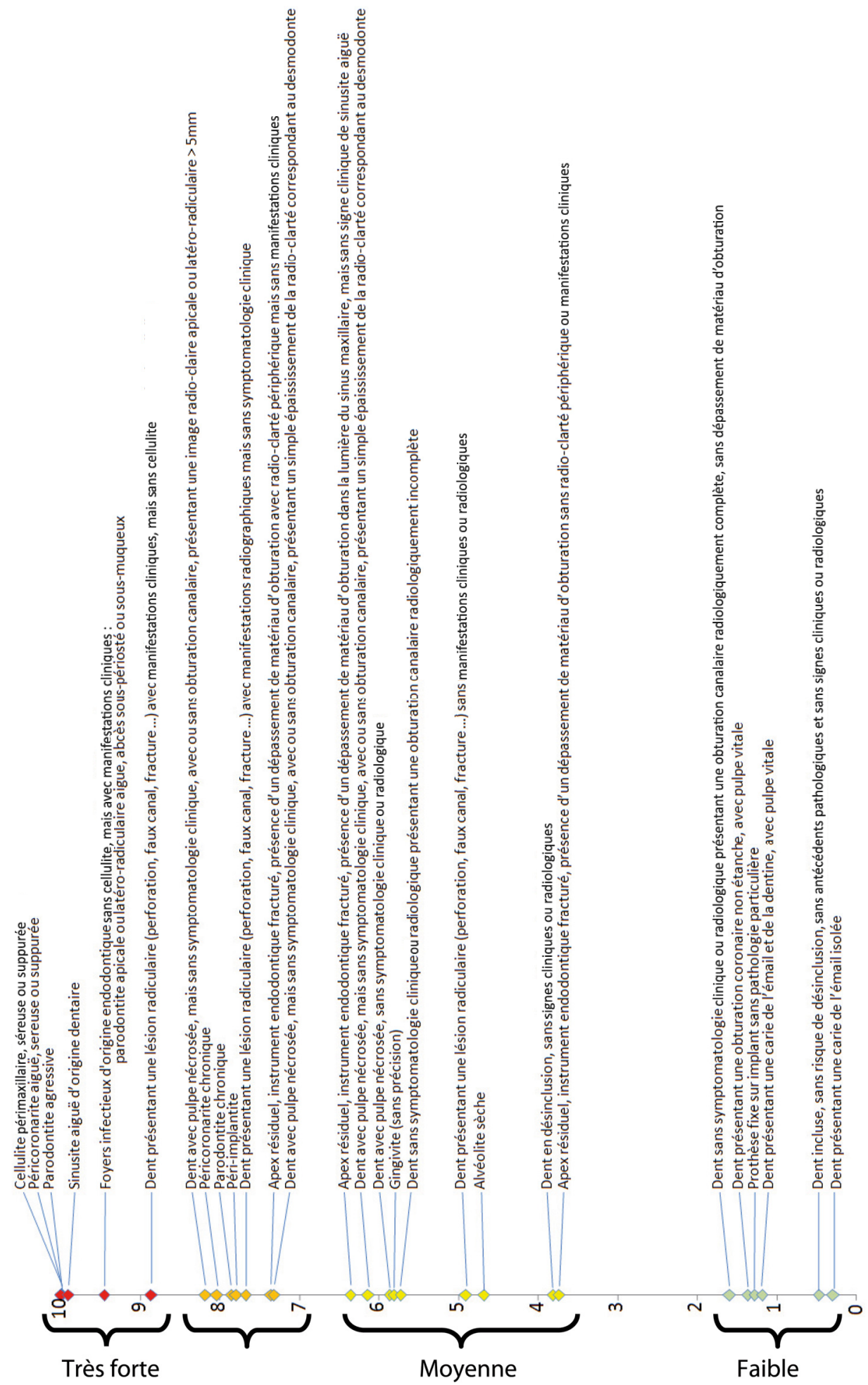

Risque infectieux supplémentaire par rapport à une dent saine sur l'arcade, de 0 à 10 . 


\section{Argumentaire}

Sommaire

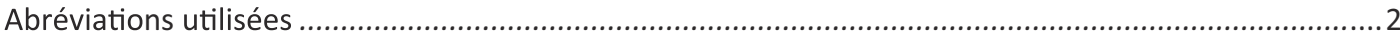

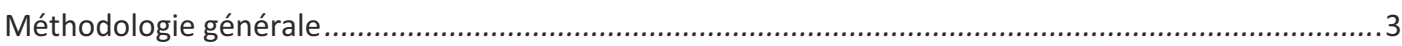

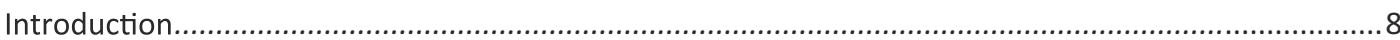

1. Définitions et physiopathologie des foyers infectieux dentaires et parodontaux ..........................9

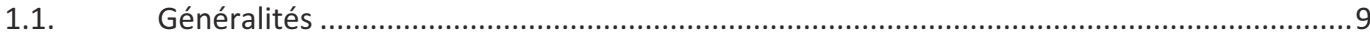

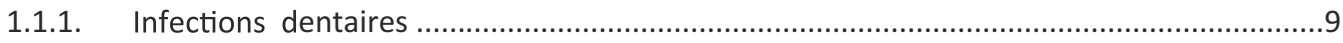

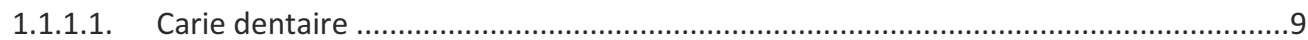

1.1.1.2. Lésions inflammatoires périradiculaires d'origine endodontique ............................10

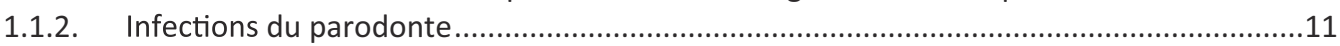

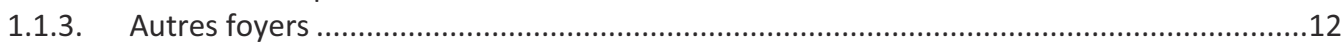

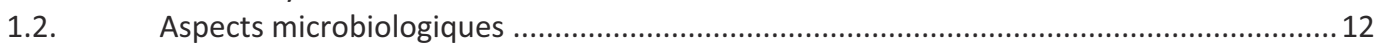

1.3. Physiopathologie du développement et de la dissémination des infections ....................15

1.3.1. Physiopathologie de la carie .................................................................................. 15

1.3.2. Physiopathologie des parodontopathies .................................................................. 15

1.3.3. Dissémination des infections ................................................................................ 16

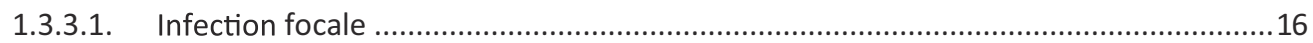

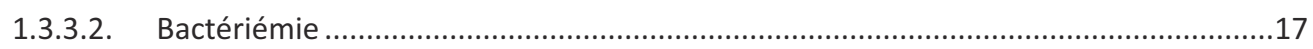

1.4. Classifications des foyers infectieux........................................................................ 18

2. Situations cliniques dans lesquelles une recherche de FIBD doit être menée................................22

2.1. Patients présentant un sepsis et/ou un syndrome inflammatoire chronique à

distance de la cavité buccale ............................................................................................................22

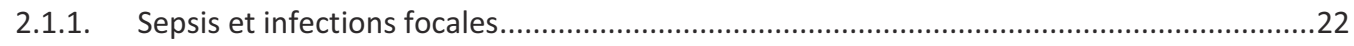

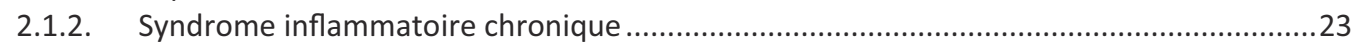

2.1.3. Pathologies immunes déclenchées ou favorisées par une infection ...............................23

2.2. Patients présentant un risque majoré d'infection bucco-dentaire du fait d'une

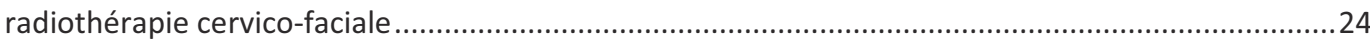

2.3. Patients susceptibles de développer une infection à point de départ bucco-

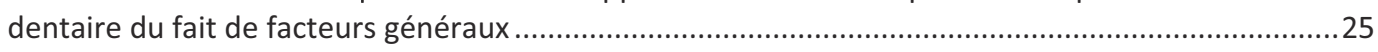

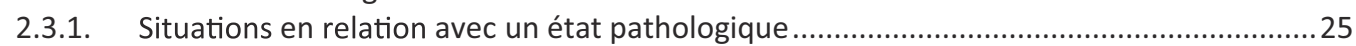

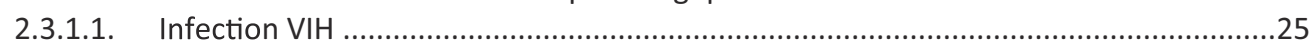

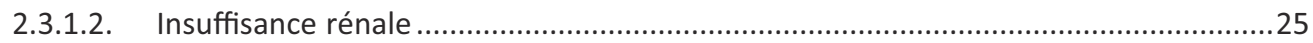

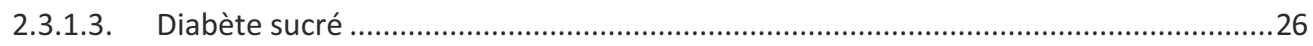

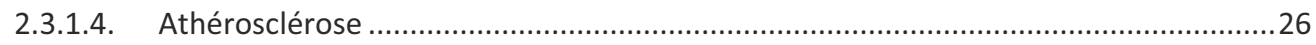

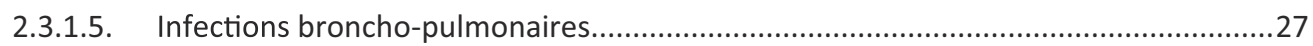

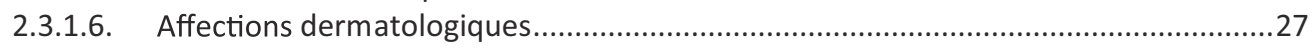

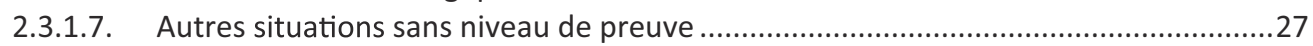

2.3.2. Situations liées à des prises médicamenteuses ............................................................28

2.3.2.1. Traitements immunosuppresseurs .......................................................................28

2.3.2.1.1. Traitements par corticoïdes ............................................................................29

2.3.2.1.2. Thérapeutiques biologiques ciblées à visée immunosuppressive .......................29

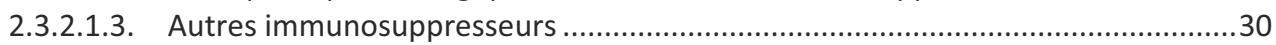




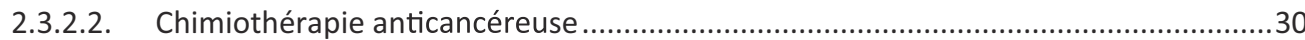

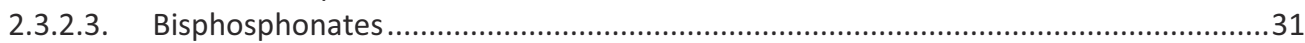

2.4. Patients susceptibles de développer une infection focale à point de départ

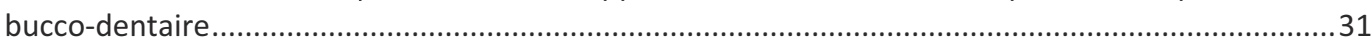

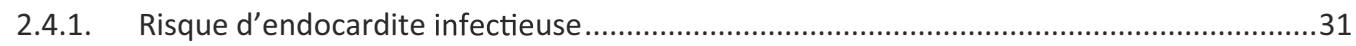

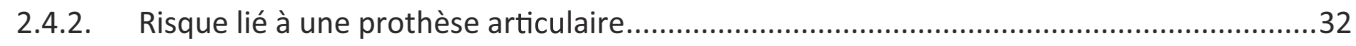

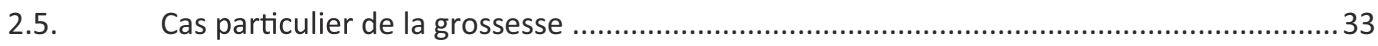

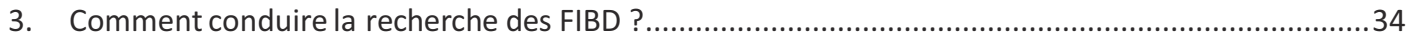

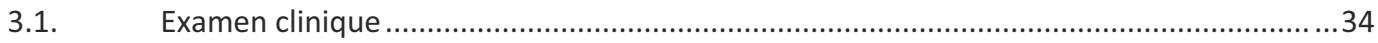

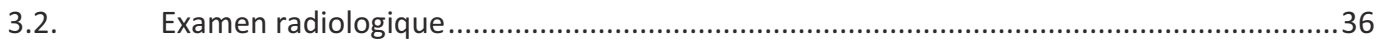

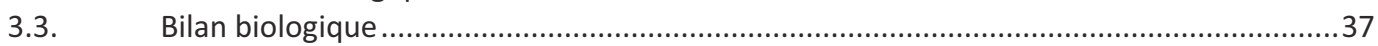

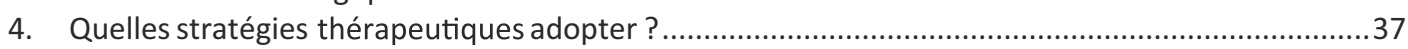

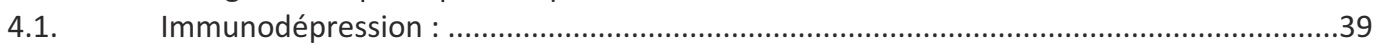

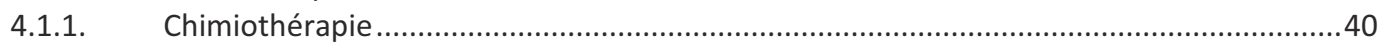

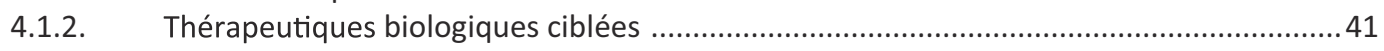

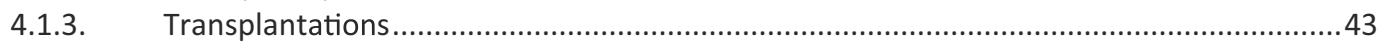

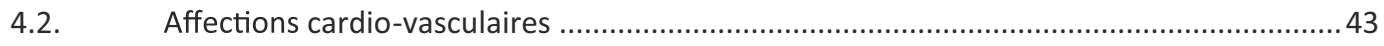

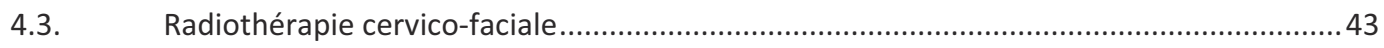

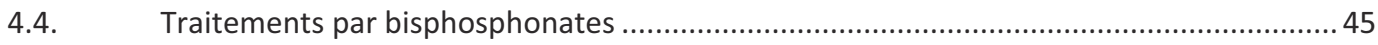

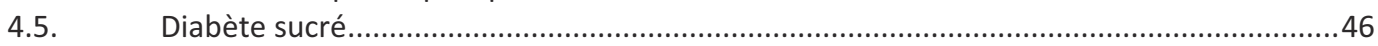

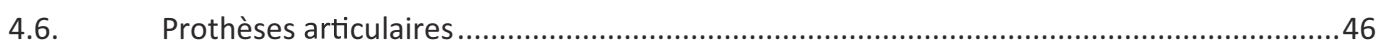

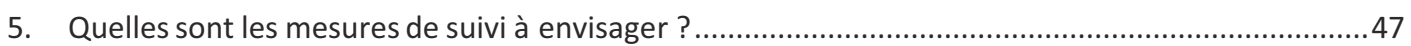

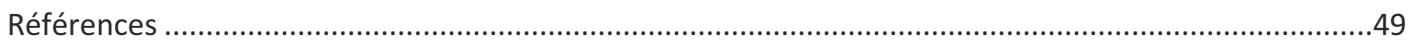

\section{Abréviations utilisées}

$\begin{array}{ll}\text { APF } & \text { Accord professionnel fort } \\ \text { CR } & \text { Consensus relatif } \\ \text { EI } & \text { Endocardite infectieuse } \\ \text { FIBD } & \text { Foyer infectieux bucco-dentaire } \\ \text { G1 } & \text { Groupe de cotateurs issus des différentes spécialités concernées } \\ \text { G2 } & \text { Groupe de cotateurs spécialistes de la cavité buccale } \\ \text { NP } & \text { Niveau de preuve } \\ \text { PC } & \text { Pas de consensus } \\ \text { SRIP } & \text { Situation à risque infectieux potentiel }\end{array}$




\section{Méthodologie générale}

\section{Objectif}

La prise en charge des foyers infectieux bucco-dentaires (FIBD) constitue une tâche quotidienne pour les spécialistes de la cavité buccale. II n'existe cependant pas de consensus pour définir les conduites à tenir, et les attitudes varient beaucoup selon l'expérience et la formation des praticiens. II s'agit pourtant d'un enjeu important en termes de santé publique. Cette situation a amené la Société Française de Chirurgie Orale (SFCO) à élaborer des recommandations destinées à éclairer les praticiens et harmoniser les pratiques.

\section{Méthodologie}

Devant le manque de ressources bibliographiques de valeur et face à des données reflétant mal l'éventail des situations rencontrées en pratique, il a été nécessaire d'adopter la méthode du consensus formalisé, codifiée par le collège de la $\mathrm{HAS}^{*}$. Conformément à cette méthodologie, le groupe de pilotage a précisé les objectifs poursuivis, les cibles des recommandations et les partenaires à associer au travail (sociétés savantes). Les questions indispensables concernant les situations cliniques retenues ont été regroupées en 4 grands axes élémentaires :

1) Quelles sont les situations cliniques dans lesquelles une recherche de FIBD doit être menée?

2) Comment conduire cette recherche?

3) Quelles stratégies adopter?

4) Quelles sont les mesures de suivi à envisager?

Après une recherche bibliographique approfondie, le groupe de pilotage a sélectionné les références pertinentes, réalisé une analyse et une synthèse critique de la littérature sous la forme d'un argumentaire bibliographique et proposé des recommandations sur la base de l'analyse de la littérature effectuée.

Deux groupes de cotation ont été constitués. Les experts du premier groupe (G1) étaient issus des différentes spécialités particulièrement concernées par le thème. Ils ont été désignés par les sociétés suivantes:

- Association Dentaire Française

- Collège National des Généralistes Enseignants

- Société Française de Cardiologie

- Société Française de Chirurgie Orale

- Société Française de Dermatologie

- Société Française d'Hématologie

- Société Française d'Ophtalmologie

- Société Française de Parodontologie et d'Implantologie Orale

- Société Française de Radiothérapie Oncologique

- Société Française de Rhumatologie

- Société Française de Stomatologie et de Chirurgie Maxillo-Faciale

- Société de Néphrologie

- Société de Pathologie Infectieuse de Langue Française

\footnotetext{
* Bases méthodologiques pour l'élaboration de recommandations professionnelles par consensus formalisé, HAS, Janvier 2006, http://www.has-sante.fr/portail/upload/docs/application/pdf/base_methodo_CFE.pdf (consulté le 12/09/2009)
} 
Le second groupe de cotateurs (G2) était constitué uniquement d'experts spécialistes de la cavité buccale : chirurgiens dentistes et stomatologues.

Tous ces cotateurs ont été choisis pour une représentativité sur la base de leur expérience professionnelle, leur mode d'exercice (hospitalo-universitaire, hospitalier, libéral) et leur origine géographique. Les travaux de ces deux groupes ont été dirigés par un président, tandis qu'un rapporteur a rédigé le document final.

Des questionnaires ont été adressés aux membres des groupes de cotation pour recueillir leur avis sur les propositions élaborées par le groupe de pilotage.

Par souci de lisibilité, dans le texte du présent argumentaire, il est fait mention du "groupe de cotation " pour désigner celui de l'un ou l'autre des deux groupes d'experts qui a procédé à la cotation pour chaque chapitre envisagé ; il est sous-entendu que le groupe G1 a statué sur les questions intéressant les diverses spécialités concernées, et le groupe G2 sur les thèmes spécifiquement buccaux.

La HAS a été tenue informée de l'avancée de ces travaux à chacun de leurs stades.

\section{Recherche documentaire}

La recherche documentaire a été effectuée par interrogation systématique des banques de données bibliographiques (Medline, Cochrane, ISI Web of knowledge et Dentistry and Oral Sciences Source) couvrant la période de janvier 1980 à avril 2011. Les langues sélectionnées ont été l'anglais et le français. Les équations de recherche ont été les suivantes :

\begin{tabular}{|c|c|c|}
\hline parodontite & \multirow{21}{*}{ et } & diabète \\
\hline parodontite apicale & & athérosclérose \\
\hline carie & & endocardite infectieuse \\
\hline cellulite & & chimiothérapie \\
\hline gingivite & & radiothérapie \\
\hline abcès dentaire & & immunodépression \\
\hline \multirow[t]{15}{*}{ infection dentaire } & & grossesse \\
\hline & & lupus érythémateux \\
\hline & & uvéite \\
\hline & & bisphosphonate \\
\hline & & anti TNF-alpha \\
\hline & & thérapeutique biologique ciblée \\
\hline & & transplantation \\
\hline & & abcès cérébral \\
\hline & & ciclosporine \\
\hline & & tacrolimus \\
\hline & & glucocorticoïde \\
\hline & & dialyse \\
\hline & & insuffisance rénale \\
\hline & & prothèse articulaire \\
\hline & & polyarthrite rhumatoïde \\
\hline
\end{tabular}




\begin{tabular}{|l|l|l|}
\hline parodontite & \multirow{5}{*}{ parodontite apicale } & bilan \\
\cline { 1 - 1 } carie & \multirow{2}{*}{ et } & prise en charge \\
cellulite & radiographie \\
\hline gingivite & orthopantomogramme \\
\hline abcès dentaire & rétro-alvéolaire \\
infection dentaire & tomodensitométrie \\
& & diagnostic \\
\hline
\end{tabular}

Ont été recherchées les recommandations pour la pratique clinique, les conférences de consensus, les articles de décision médicale, les revues de littérature, les méta-analyses et autres travaux d'évaluation déjà publiés au plan national et international.

En vue de sélectionner des articles non identifiés lors de l'interrogation, les références citées dans les articles analysés ont aussi été recherchées.

De plus, ont été consultés les textes législatifs et réglementaires pouvant avoir un rapport avec le thème, de même que les sites Internet utiles (agences gouvernementales, sociétés savantes) ainsi que les articles des fonds bibliographiques des membres du groupe de pilotage et des groupes de cotation. Parmi les références qui ont été sélectionnées et analysées, 143 ont été utilisées pour l'élaboration du texte.

Chaque article a été analysé en appréciant la qualité méthodologique des études, afin d'affecter à chacun un niveau de preuve scientifique. Les recommandations ont été classées selon leur force en tenant compte du niveau de preuve des études sur lesquelles elles s'appuient ${ }^{*}$.

\begin{tabular}{|c|c|c|}
\hline $\begin{array}{l}\text { Niveau de } \\
\text { preuve } 1\end{array}$ & $\begin{array}{l}\text { - } \text { Essais comparatifs randomisés de forte puissance } \\
\text { - } \quad \text { Méta-analyse d'essais comparatifs randomisés } \\
\text { - Analyse de décision basée sur des études bien menées }\end{array}$ & Preuve scientifique établie \\
\hline $\begin{array}{l}\text { Niveau de } \\
\text { preuve } 2\end{array}$ & $\begin{array}{l}\text { - Essais comparatifs randomisés de faible puissance } \\
\text { - Études comparatives non randomisées bien menées } \\
\text { - Études de cohorte }\end{array}$ & Présomption scientifique \\
\hline $\begin{array}{l}\text { Niveau de } \\
\text { preuve } 3\end{array}$ & - Études cas-témoins & \multirow{2}{*}{$\begin{array}{l}\text { Faible niveau de preuve } \\
\text { scientifique }\end{array}$} \\
\hline $\begin{array}{l}\text { Niveau de } \\
\text { preuve } 4\end{array}$ & $\begin{array}{l}\text { - Études comparatives comportant des biais importants } \\
\text { - Études rétrospectives } \\
\text { - Séries de cas }\end{array}$ & \\
\hline
\end{tabular}

\section{Rédaction des propositions de recommandations}

Les données issues de l'analyse critique et leur synthèse ont été discutées en séance au sein du groupe de pilotage, qui a établi les premières propositions de recommandations. L'ensemble de ces propositions, qu'elles aient été ou non fondées sur un niveau de preuve élevé, ont été soumises aux groupes de cotation. Dans le texte final, les recommandations fondées sur les niveaux de preuve les

\footnotetext{
* Guide d'analyse de la littérature et gradation des recommandations, ANAES, janvier 2000

http://www.has-sante.fr/portail/upload/docs/application/pdf/analiterat.pdf (consulté le 12/09/2009)
} 
plus élevés ont été clairement différenciées des propositions de recommandations fondées sur le seul consensus professionnel.

\section{Cotation}

Deux phases de cotation ont eu lieu successivement pour chaque groupe de cotation.

La première phase de cotation s'est accompagnée de l'envoi de l'argumentaire bibliographique et des propositions de recommandations rédigées par le groupe de pilotage.

Pour chaque proposition, les membres des groupes de cotation devaient donner une réponse allant de 1 (totalement inappropriée) à 9 (totalement appropriée). La cotation était fondée sur la synthèse des données publiées dans la littérature (jointe au questionnaire et dont le but était d'informer sur l'état des connaissances publiées) et/ou sur l'expérience du cotateur dans le domaine abordé.

Lors de l'analyse des résultats du premier tour de cotation, toutes les réponses ont été prises en compte. En cas de valeurs manquantes lors des cotations, les cotateurs concernés ont été contactés individuellement afin de leur demander de se positionner.

L'étalement des réponses a permis de définir s'il y avait accord ou désaccord entre les membres du groupe sur une proposition donnée. En cas d'accord entre les membres du groupe, l'analyse des réponses a permis également de préciser la force de l'accord. Ainsi si l'intervalle des réponses était situé à l'intérieur des bornes d'une seule des 3 zones [1 à 3] ou [4 à 6] ou [7 à 9], il existait un accord «fort » entre les membres du groupe de cotation sur le caractère approprié de l'intervention, sur son caractère inapproprié ou sur une indécision quant à son caractère approprié. Si l'intervalle des réponses empiétait sur la zone voisine, il existait un accord qualifié de "relatif » entre les membres du groupe de cotation. En cas d'étalement des réponses sur l'ensemble des 3 zones ou de réponses comprises dans les 2 zones extrêmes [1 à 3] et [7 à 9], il existait un désaccord entre les membres du groupe de cotation sur le caractère approprié d'une proposition.

Dans le texte, les résultats sont exprimés en utilisant les abréviations suivantes :

\begin{tabular}{|c|c|}
\hline APF & Accord professionnel fort \\
\hline CR & Consensus relatif \\
\hline PC & Pas de consensus \\
\hline
\end{tabular}

A l'issue de la première phase de cotation, le groupe de cotation a été réuni pour une discussion autour des propositions de recommandations et de l'argumentaire bibliographique qui les soustendait.

La seconde phase de cotation a été réalisée après cette réunion par envoi en courrier individuel d'un document et d'un questionnaire modifiés en fonction de la discussion menée en séance lors de la réunion.

Lors de l'analyse des résultats du second tour de cotation, un degré de tolérance dans la définition de l'accord et de sa force a été accepté dans la mesure où deux des réponses extrêmes, l'une minimale et l'autre maximale, ont pu être écartées.

Les propositions de recommandations n'ont été retenues que s'il n'existait au maximum qu'une seule réponse à l'extrême opposé de la zone où se situait la médiane des réponses.

Il a été procédé de la même façon dans chacun des deux groupes de cotation. 


\section{Formulation des recommandations}

À l'issue de l'analyse des réponses des groupes de cotation, le groupe de pilotage a formulé les recommandations en fonction de ces réponses et finalisé les documents à adresser au groupe de lecture (ainsi qu'aux groupes de cotation pour information).

Les rédacteurs n'ont pas fait apparaître dans la synthèse les cas pour lesquels aucun consensus n'a été dégagé, hormis les situations pour lesquelles ils estimaient important que cette absence de consensus soit connue.

\section{Contrôle des recommandations}

Les recommandations ont été présentées et discutées en assemblée le 21 mai 2011 lors de la séance scientifique de la Société Française de Chirurgie Orale à Nantes.

Le texte des recommandations et de l'argumentaire a ensuite été soumis à un groupe de lecture, extérieur aux groupes de cotation, avant d'être définitivement finalisé. Le groupe de lecture était composé d'experts choisis notamment pour la diversité de leur compétence (cardiologie, chirurgie générale, chirurgie maxillo-faciale, chirurgie vasculaire et thoracique, dermatologie, infectiologie, médecine générale, néphrologie, odontologie, oncologie, ophtalmologie, oto-rhino-laryngologie, radiothérapie, rhumatologie, stomatologie), de leur mode d'exercice (hospitalo-universitaire, hospitalier, libéral) et de leur origine géographique. Les experts du groupe de lecture étaient chargés de donner un avis argumenté sur la qualité méthodologique et la validité scientifique du texte proposé. Les remarques du groupe de lecture ont été transmises aux groupes de cotation pour modification du texte et validation du document final.

\section{Contrôle de la validité de la phase de cotation}

Trois membres du groupe de pilotage ayant par erreur été appelés à participer à la phase de cotation, une analyse approfondie de l'impact de leur présence sur chaque cotation a été effectuée à la demande du chef de projet de la HAS. Cette analyse a montré que la présence des membres du groupe de pilotage n'avait aucune incidence sur le résultat des cotations.

Le financement de ces recommandations a été assuré exclusivement par la Société Française de Chirurgie Orale, avec le soutien logistique de l'Association Dentaire Française. 


\section{Introduction}

La pathologie infectieuse de la cavité buccale constitue un domaine particulièrement vaste qui motive une proportion considérable des consultations. Les caries et les parodontopathies représentent les situations les plus fréquentes, davantage que les atteintes de la muqueuse buccale, qu'elles soient fungiques, bactériennes ou virales. A côté de ces préoccupations quotidiennes strictement buccales, le spécialiste de la cavité buccale reçoit également des patients adressés par des praticiens de différentes spécialités pour une recherche de foyers infectieux bucco-dentaires (FIBD).

La recherche de FIBD est essentiellement demandée par le correspondant dans quatre circonstances :

- en raison d'un état général particulier, soit chez les patients pour lesquels l'apparition d'une infection secondaire est à craindre (diabète sucré, SIDA, patients à risque d'endocardite infectieuse), soit dans le but de stabiliser une pathologie générale (diabète sucré, polyarthrite rhumatoïde) ;

- pour rechercher le point de départ d’une infection secondaire (abcès cérébral, endocardite, sepsis) ;

- avant d'instaurer une thérapeutique médicale ou physique suivie par un patient, susceptible de favoriser ou d'aggraver un processus infectieux (chimiothérapie anticancéreuse, radiothérapie, traitements immunosuppresseurs, traitements par bisphosphonates...);

- pour préparer le patient à une intervention chirurgicale (prothèses valvulaires, endoprothèses, prothèses articulaires...) ou encore à la suite d'une telle intervention.

Les FIBD peuvent en effet aggraver ou déstabiliser certaines pathologies (comme par exemple le diabète sucré ou la polyarthrite rhumatoïde). A l'inverse, les états pathologiques diminuant les défenses de l'organisme sont susceptibles de réactiver des infections buccales. Enfin, les FIBD, même minimes, peuvent avoir à distance des répercussions importantes sur les différents appareils de l'organisme : c'est ce concept qui a été présenté au début du siècle dernier sous le terme d'infection focale.

La recherche des FIBD à visée prophylactique ou curative revêt une importance toute particulière chez les malades présentant un risque infectieux majeur (risque d'endocardite, transplanté, immunodéprimé), ou une pathologie d'étiologie inconnue (fièvre au long cours inexpliquée, septicémie).

L'ensemble des pathologies infectieuses de la cavité buccale est concerné par ces problèmes. Cependant, devant l'ampleur du domaine, le groupe de pilotage a délibérément choisi de limiter le champ de ces recommandations aux cas les plus fréquents, c'est-à-dire

- d'une part aux seules manifestations infectieuses d'origine bactérienne (sont donc exclues $a$ priori les pathologies fungiques ou virales)

- et d'autre part aux seuls foyers infectieux dentaires et parodontaux : sont donc exclues $a$ priori les autres pathologies infectieuses muqueuses (stomatites, angines...), osseuses d'origine non dentaire et salivaires. 


\section{Cibles de ces recommandations}

Ce travail a été conçu notamment pour aider les praticiens spécialistes de la cavité buccale isolés des structures hospitalières, à gérer des situations pratiques. II s'adresse aussi aux cardiologues, dermatologues, endocrinologues, hématologues, infectiologues, médecins généralistes, néphrologues, oncologues, radiothérapeutes, rhumatologues, chirurgiens orthopédistes, thoraciques, vasculaires, et d’une façon générale aux praticiens de toutes les spécialités concernées par ces problèmes.

C'est justement à l'intention des praticiens qui ne sont pas spécialistes de la cavité buccale qu'un chapitre sur les définitions et la physiopathologie des foyers infectieux dentaires et parodontaux a été rédigé au début de ce document. Les notions qui y sont développées paraitront nécessairement élémentaires aux spécialistes de la cavité buccale. II en va de même pour le chapitre sur la pratique de l'examen buccal.

Enfin, le titre de ce document évoque les foyers infectieux "bucco-dentaires ». Cette expression usuelle a été retenue pour des raisons de lisibilité, afin de ne pas dérouter ou rebuter les praticiens qui ne seraient pas spécialistes de la cavité buccale. En réalité, il sera uniquement traité dans ce texte des foyers infectieux dentaires et/ou parodontaux.

\section{Définitions et physiopathologie des foyers infectieux dentaires et parodontaux}

\subsection{Généralités}

Ce travail s'adressant à des praticiens de nombreuses spécialités médicales, il est apparu nécessaire au groupe de pilotage de rappeler le cadre physiopathologique des infections dentaires et parodontales.

Les foyers infectieux dentaires et parodontaux sont associés à une flore bactérienne spécifique ; ainsi, certains micro-organismes pathogènes sont impliqués dans la maladie carieuse, les infections de l'endodonte et les parodontopathies. Les lésions d'origine endodontique ou parodontale sont reconnues comme étant deux étiologies majeures parmi les infections primaires [1]. La littérature indique que de nombreux micro-organismes caractéristiques de la flore buccale sont responsables d’infections à distance et de maladies générales.

\subsubsection{Infections dentaires \\ 1.1.1.1. Carie dentaire}

La carie dentaire est une maladie infectieuse, polymicrobienne et multifactorielle. Elle résulte de l'interaction entre l'hôte, les bactéries cariogènes du biofilm dentaire et les habitudes alimentaires. Cliniquement, elle se traduit par la lésion carieuse, c'est-à-dire la destruction progressive des tissus durs de la dent (émail, dentine, cément). Un grand nombre d'espèces bactériennes est retrouvé dans les caries profondes et les caries radiculaires. Toutefois, les bactéries principalement retrouvées sont les streptocoques, les lactobacilles et les actinomyces. 


\subsubsection{Lésions inflammatoires périradiculaires d'origine endodontique}

Il s'agit de pathologies infectieuses intéressant l'endodonte avec une participation du péri-apex; elles peuvent être aiguës ou chroniques, symptomatiques ou asymptomatiques, associées ou non à une lésion péri-apicale ou latéro-radiculaire.

L'infection endodontique primaire (nécrose pulpaire) est une infection polymicrobienne. Elle est caractérisée par une grande variété de combinaisons bactériennes : en moyenne 4 à 7 espèces, surtout anaérobies, comprenant des bactéries à Gram positif et à Gram négatif. Cette flore varie selon la localisation endocanalaire et s'enrichit progressivement de bactéries à Gram négatif et de bactéries anaérobies (« dérive anaérobie »).

Infections périapicales primaires

Il peut s'agir de pathologies aiguës ou chroniques, symptomatiques ou non, avec ou sans complications péri-maxillaires.

On retrouve:

- Des pathologies aiguës

- desmodontite apicale aiguë (également appelée parodontite apicale aiguë, ou parodontite apicale symptomatique)

- abcès apical aigu: dent infectée symptomatique sans complication périmaxillaire (desmodontite aiguë suppurée, parodontite péri-apicale aiguë suppurée, abcès sous périosté ou sous-muqueux)

- cellulite séreuse ou suppurée: dent infectée symptomatique avec complication périmaxillaire

- Des pathologies chroniques

- nécrose asymptomatique (nécrobiose)

- nécrose asymptomatique avec lésion apicale (granulome apical, kyste radiculo-dentaire, parodontite apicale chronique)

- desmodondite péri-apicale chronique suppurée

- parodontite apicale récurrente

Quel que soit leur type, les lésions périapicales sont septiques. La flore endocanalaire des dents présentant des lésions apicales est souvent identique à celle des abcès, des granulomes et des kystes. Elle est composée de $87 \%$ d'espèces anaérobies.

La symptomatologie serait liée à la nature de l'écosystème des canaux infectés; par exemple dans le cas d'une dent asymptomatique, il est majoritairement composé de bactéries anaérobies strictes (64 à 87 \%) (Porphyromonas et Prevotella). 
Infections endodontiques et périapicales secondaires

II s'agit d'infections périapicales intéressant des dents qui ont fait l'objet d'un traitement endodontique. Ces infections sont dues soit à des bactéries initialement présentes dans le canal infecté et qui persistent malgré le traitement canalaire chimio-mécanique, soit à des bactéries qui étaient absentes des canaux infectés et qui ont pénétré le système endocanalaire à l'occasion d'un geste thérapeutique initial, soit encore par voie hématogène. La flore est peu abondante et elle est dominée par des bactéries anaérobies facultatives à Gram positif (Actinomyces, Enterococcus et Streptococcus).

\subsubsection{Infections du parodonte}

Les maladies parodontales ou parodontopathies peuvent être définies comme des maladies infectieuses multifactorielles. Ces foyers sont souvent sous-estimés. Pour Roth [2], les foyers parodontaux sont plus dangereux que les foyers apicaux étant donné que la surface de la lésion est plus importante.

Les parodontopathies peuvent se manifester par une inflammation visible ou non, des saignements gingivaux spontanés ou provoqués d'importance variable, la formation de poches en rapport avec des pertes d'attache et d'os alvéolaire, une mobilité dentaire, et peuvent conduire à la perte de dents.

Le passage d'un état gingival sain à une gingivite, puis à une parodontite suit le phénomène de " dérive anaérobie »[3].

Gingivite chronique

C'est une inflammation d'origine microbienne du parodonte superficiel sans atteinte du parodonte profond. Elle est liée au manque d’hygiène et à une accumulation de plaque dentaire et de tartre.

\section{Parodontite}

C'est une inflammation du parodonte profond d'origine microbienne. Elle s'accompagne d'une perte d'attache épithélio-conjonctive, d'une alvéolyse ayant pour conséquence une dénudation radiculaire pouvant aller jusqu'à la perte de la dent. La flore est essentiellement composée de bactéries anaérobies, à Gram négatif et de spirochètes.

On distingue plusieurs types de parodontites qui diffèrent par leurs signes cliniques, radiologiques, leur terrain et leur microbiologie :

- parodontite chronique

- parodontite agressive

- parodontites associées à des maladies générales ou infectieuses

- parodontite réfractaire

- parodontite ulcéro-nécrotique. 


\subsubsection{Autres foyers}

Dents en désinclusion et péricoronarite

Une dent en désinclusion est une dent en cours d'éruption, dont la couronne est en communication avec le milieu buccal, suite à l'ouverture du sac péricoronaire.

Une péricoronarite est une atteinte inflammatoire du sac péricoronaire. Elle peut être aiguë, congestive ou suppurée, ou chronique.

«Péri-implantite »

Les implants dentaires ont, à la différence des implants orthopédiques, une partie transmuqueuse en rapport avec le milieu extérieur de la cavité buccale. Un biofilm se forme sur les pièces transmuqueuses. Sur ce biofilm peut se développer une infection susceptible de détruire les tissus péri-implantaires et d'entrainer à terme la perte de l'implant. On parle également de mucosite périimplantaire [4] (inflammation réversible, localisée aux tissus mous superficiels) ou de "périimplantite » (processus inflammatoire destructif) en fonction de l'atteinte des tissus autour des implants ostéo-intégrés [5].Ces lésions peuvent former une poche péri-implantaire.

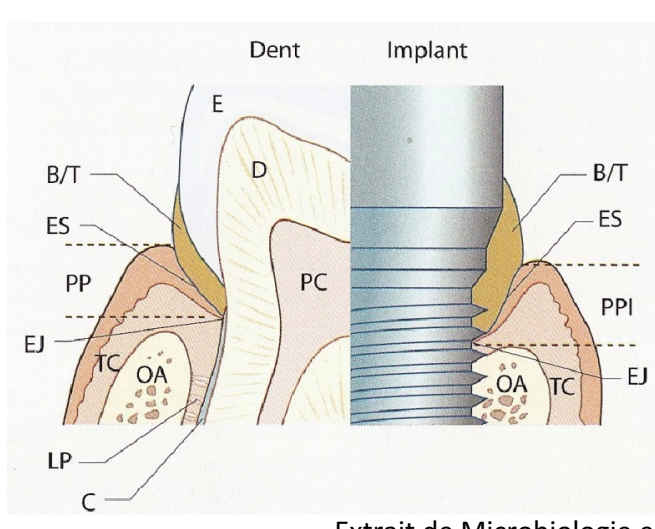

Comparaison du développement d'une parodontite et d'une parodontite péri-implantaire.

$\mathrm{E}=$ émail

$D=$ dentine

$\mathrm{PC}=$ pulpe camérale

$C=$ cément

$\mathrm{LP}=$ ligament parodontal

$\mathrm{OA}=$ os alvéolaire

$\mathrm{TC}=$ tissu conjonctif

$E J$ = épithélium de jonction

ES = épithélium sulculaire

$\mathrm{PP}=$ poche parodontale

$\mathrm{PPI}=$ poche péri-implantaire

$\mathrm{B} / \mathrm{T}=$ biofilm/tartre

Extrait de Microbiologie en odontostomatologie (Fig. 3.41).

Chardin H, Barsotti O, Bonnaure-Mallet M. Maloine, Paris, 2006.

\subsection{Aspects microbiologiques}

Microbiologie des infections bucco-dentaires (d'après l'AFSSAPS) [6].

La flore buccale constitue un écosystème complexe riche en bactéries et composé de plus de 500 espèces, réparties en environ 20 genres bactériens. Cette flore varie dans le temps mais aussi d'un site de prélèvement à l'autre et parfois selon les individus [7].

Dès la naissance, la flore buccale, inexistante in utero, se constitue à partir de l'environnement, et principalement au contact de la mère lors du maternage. Des bactéries sont présentes 
transitoirement, mais certaines d'entre elles colonisent de façon durable la cavité buccale de l'enfant en adhérant grâce à des récepteurs spécifiques. A ce stade, l'immaturité immunologique autorise cette première colonisation.

Ensuite, la première denture augmente significativement le nombre potentiel de niches et sites de fixation des bactéries, de même que le sillon gingival permet d'autres colonisations en atmosphère anaérobie.

Durant les premières années de la vie, la flore buccale est sans cesse remaniée jusqu'à l'apparition de la denture permanente. La salive et le fluide gingival assurent l'apport de nutriments nécessaires à la croissance des bactéries. Ils transportent également des enzymes et des anticorps qui vont inhiber l'adhésion et la croissance des micro-organismes.

Les bactéries n'adhèrent pas seulement aux surfaces : elles sont capables de former des co-agrégats. C'est ainsi que la colonisation initiale par les streptocoques (S. salivarius, S. mitis) est suivie d'une coagrégation avec des actinomycètes (Actinomyces odontolyticus) pour constituer la pellicule exogène acquise à la surface des dents sur laquelle d'autres bactéries pourront alors se fixer (Fusobacterium nucleatum) créant de nouvelles niches de survie pour d'autres bactéries anaérobies strictes qui se retrouvent dans ce milieu aéré. Une cascade de colonisations successives aboutira à la constitution d'un biofilm oral de plus en plus complexe. Celui-ci évolue tout au long de la vie.

En 1995, Elder et al. [8] ont proposé une revue des différents comportements des microorganismes et de leur propension à s'organiser en biofilms face à des conditions d'environnement moins favorables.

S'il était admis qu'il existe un biofilm parodontal largement variable suivant l'état de santé du parodonte et différent du biofilm recouvrant la dent dans sa partie intra-buccale, c'est plus récemment qu'a été admise l'existence d'un biofilm endodontique, pulpaire, sur les dents ayant perdu leur vitalité [9]. Les mêmes conditions y sont présentes qu'autour des diverses endoprothèses humaines: des produits de substitution remplacent une structure naturelle et forment le lit du développement d'un biofilm. De plus, cette production a lieu dans des conditions d'anaérobiose relativement strictes, voire totales, et à distance des moyens de défense de l'organisme, c'est-à-dire dans les conditions optimales pour l'apparition et l'extension d'un biofilm.

Debelian et al. [10] ont démontré que tous les canaux endodontiques sans réaction apicale symptomatique contiennent des micro-organismes (Fusobacterium nucleatum, Prevotella intermedia, Propionibacterium acnes, Propionibacterium propionicus, Peptostreptococcus anaerobius, Eubacterium sp., Porphyromonas endodontalis, Staphylococcus aureus...), avec une nette prédominance de microorganismes anaérobies. De plus, lors d'une intervention endodontique orthograde sur ce type de dent, qu'elle passe au delà ou se termine à $2 \mathrm{~mm}$ en deçà du foramen apical, une bactériémie est observée dans 33 à $50 \%$ des cas.

Leonardo et al. [11] ont mis en évidence que les dents présentant une nécrose pulpaire et une lésion péri-apicale chronique, ainsi que les dents présentant une infection persistante ou résistante aux thérapies, contiennent des micro-organismes dans l'entièreté de leur système canalaire, depuis le canal principal jusqu'aux tubuli dentinaires, en passant par les canaux latéraux, secondaires et accessoires, les ramifications du delta apical, le foramen apical, ainsi que les aires de résorption du cément apical et les tissus péri-apicaux. Les régions de résorption du cément, adjacentes au foramen 
apical, sont envahies par de nombreux micro-organismes, soit seuls (coques, bacilles ou filaments) soit en association (bacilles et filaments).

En cas de parodontite, la flore de la poche parodontale contient une plus grande proportion de bacilles à Gram négatif. Parmi ces bacilles, Aggregatibacter actinomycetemcomitans, Porphyromonas gingivalis et Prevotella intermedia sont considérés comme les trois principales bactéries responsables de ces pathologies. Ces pathogènes sont absents de la flore buccale avant l'âge de 3 ans. La colonisation se fait à partir des contacts du sujet avec d'autres individus. On trouve plus fréquemment ces pathogènes dans la flore d'enfants appartenant à des familles affectées par une parodontite. Cette contamination semble assez tardive et survient peu dans l'enfance, sauf pour Aggregatibacter actinomycetemcomitans qui commence à coloniser l'enfant vers 4 à 7 ans. Les progrès taxonomiques ont permis de montrer qu'il s'agit d'un groupe hétérogène. Si Prevotella pallens et Prevotella nigrescens sont retrouvées chez le jeune enfant, il n'en est pas de même de Prevotella intermedia stricto sensu désormais considérée comme parodontopathogène. Lors de la colonisation initiale, on observe une très grande hétérogénéité clonale des souches pionnières. On sait qu'une transmission mère-enfant est fréquente, mais dans certaines situations pathologiques l'origine du pathogène demeure inconnue. Si l'adhésion est l'étape majeure de la colonisation, on ignore quels sont les facteurs qui permettent la persistance de certains clones. Tannerella forsythensis, isolé plus récemment, est considéré comme un autre agent parodontopathogène.

La pathologie clinique dentaire et péri-dentaire résulte d'interactions complexes entre l'hôte et un micro-environnement écologique.

L'importance de l'organisation bactérienne en biofilm étant prouvée, il faut compléter cette notion d'organisation spatiale par une notion d'organisation qualitative. Les relations interbactériennes ne sont en effet pas le fruit du hasard. Socransky et al. [12], en 1998, a montré que les espèces bactériennes impliquées dans les pathologies parodontales pouvaient être rassemblées par groupes. La notion de complexes bactériens dans la flore parodontopathogène prend forme : il n'est plus possible de parler de pathogénie parodontale associée à une seule bactérie, hormis pour Aggregatibacter actinomycetemcomitans.

On retrouve donc:

- Aggregatibacter actinomycetemcomitans sérotype b qui forme un complexe à lui seul, n'ayant pas pu être rapproché des autres bactéries ;

- le complexe jaune formé de Streptococcus gordonii, Streptococcus intermedius, Streptococcus mitis et Streptococcus sanguis

- le complexe vert de Capnocytophaga spp., Aggregatibacter actinomycetemcomitans sérotype a, Eikenella corrodens et Campylobacter concisus ;

- le complexe violet de Veillonella parvula et Actinomyces odontolyticus ;

- le complexe orange de Campylobacter gracilis, Campylobacter rectus, Campylobacter showae, Eubacterium nodatum, Prevotella intermedia, Prevotella nigrescens, Peptostreptococcus micros, et des sous-espèces de Fusobacterium nucleatum ;

- le complexe rouge de Porphyromonas gingivalis, Tannerella forsythensis et Treponema denticola. 


\subsection{Physiopathologie du développement et de la dissémination des infections}

On distingue deux processus physiopathologiques différents : les pathologies engendrées par la carie et les pathologies parodontales.

\subsubsection{Physiopathologie de la carie}

Carie superficielle

La carie superficielle, limitée à la dentine, engendre d'abord une sensibilité aux agressions thermiques et chimiques (essentiellement l'acide et le sucre, surtout dans ses formes les plus collantes : bonbons, chocolat, miel). Cette sensibilité, de plus en plus intense et durable avec la profondeur de l'atteinte carieuse, est toutefois toujours limitée à la durée de l'application de la cause déclenchante. Elle est retrouvée à l'examen par l'aspersion des dents à l'eau froide ou chaude.

Pulpite

La pulpite se distingue par l'intensité de la douleur : c'est la classique " rage de dent »; la douleur est provoquée, mais cette fois par des variations thermiques minimes : la chaleur de l'oreiller ou la simple aspiration de l'air ambiant. Elle persiste après l'application de la cause déclenchante et ne s'atténue que lentement. Elle est le plus souvent précédée de douleurs provoquées au cours des repas et négligées ; la pulpite est immédiatement soulagée par l'excision de l'essentiel du paquet vasculonerveux présent dans la chambre pulpaire et le système endo-canalaire.

Nécrose pulpaire et desmodontite

Quelquefois, après un stade fugace de pulpite aiguë, la lésion pulpaire évolue vers la nécrose. D'abord silencieuse, la nécrose peut ensuite être responsable, avec l'installation d'une infection à microorganismes anaérobies, d'une "desmodontite ": la douleur est provoquée par la pression ou la percussion sur la dent. L'élargissement inflammatoire du ligament desmodontal entraîne une légère égression de la dent vers le plan occlusal et donc un contact prématuré sur la dent concernée. Ce contact, très douloureux, est inévitable, par exemple lors de la déglutition automatique de la salive qui entraîne l'occlusion dentaire. L'aspersion à l'eau froide est muette ou même calme la douleur, mais le chaud, au contraire, l'exagère.

Pathologie apicale

L'infection peut encore s'aggraver soit d'emblée, soit après une phase chronique pendant laquelle se constitue un granulome apical, dont le réchauffement aboutit à l'abcès dentaire. La douleur devient alors permanente, lancinante, tandis que la clinique s'enrichit des signes de l'infection maxillaire et périmaxillaire : la tuméfaction et la fièvre.

\subsubsection{Physiopathologie des parodontopathies}

L'agression bactérienne constitue le premier facteur indispensable de développement de la maladie parodontale. 
Le tartre est formé par la plaque dentaire minéralisée. L'inflammation est liée à l'irritation locale due au tartre, qui constitue un support idéal pour la flore pathogène buccale. L'inflammation et les bactéries provoquent une protéolyse, entraînant une fragilisation et une destruction plus ou moins localisée de la gencive.

La parodontite progresse par phases cycliques d'activité et de repos. La présence de poches parodontales n'est pas considérée comme un indicateur de l'activité de la maladie, mais représente plutôt la somme de la destruction parodontale engendrée par les périodes d'exacerbation passées. La cyclicité observée serait en étroite relation avec l'efficacité de la réponse immunitaire de l'hôte.

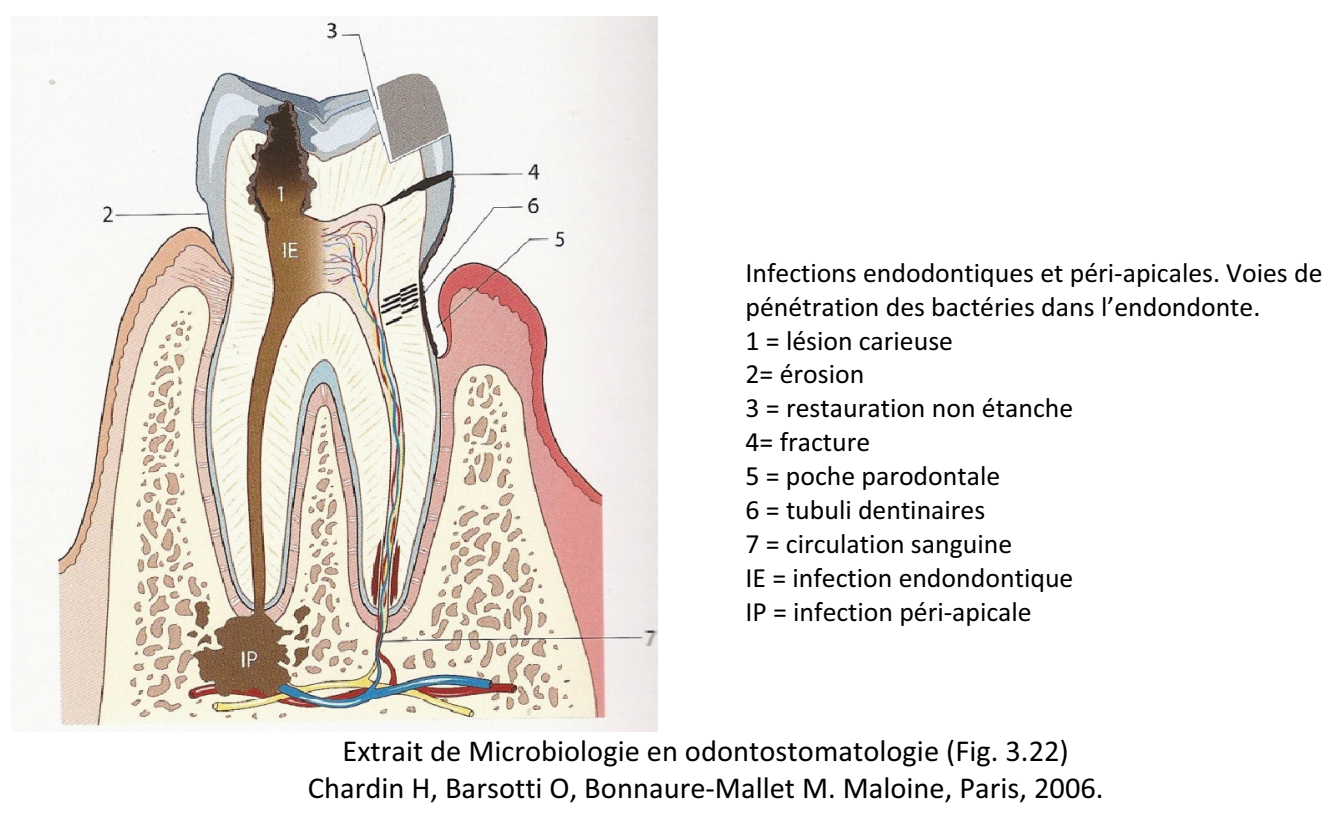

\subsubsection{Dissémination des infections}

\subsubsection{Infection focale}

Historiquement, la notion d'infection focale d'origine buccale est apparue avec Miller en 1891. Billings en 1912, a tenté de démontrer un lien entre des cas d'arthrite chronique et une infection orale.

La théorie de Thoden van Velzen [13] suggérait trois mécanismes possibles responsables des foyers à distance :

1) l'infection métastatique : le foyer secondaire est causé par la colonisation à distance d'un terrain favorable au développement des micro-organismes présents dans le foyer primaire, généralement chez un individu fragilisé.

2) un foyer métastatique " dû aux toxines microbiennes "; ce sont les exo- ou endotoxines libérées par les micro-organismes qui provoqueraient des réactions plaquettaires, et pourraient entraîner un envahissement tissulaire.

3) une "inflammation métastatique » due "au traumatisme immunologique »: les antigènes solubles libérés entraîneraient la formation de complexes immuns se déposant dans les tissus. 


\subsubsection{Bactériémie}

La bactériémie traduit la présence de bactéries dans le sang circulant. Le passage des bactéries dans la circulation sanguine ou lymphatique peut résulter d'une action mécanique.

Les soins dentaires représentent théoriquement des situations à risque de contamination. Le nombre de bactéries qui entrent dans la circulation sanguine lors des traitements dentaires est estimé à 1 à 10 bactéries par $\mathrm{ml}$ de sang (ou CFU/ml), avec une chute de ce taux de 10 à $15 \%$ après 10 minutes. Les micro-organismes ainsi disséminés dans l'organisme sont rapidement détruits par le système immunitaire. Néanmoins, dans des conditions défavorables, cette bactériémie peut conduire à une infection focale.

La fréquence des bactériémies après soins dentaires est très variable en fonction des auteurs et des gestes réalisés. Pour Okabe et al. [14], dans une étude pourtant sur 183 patients, une bactériémie était retrouvée dans $72 \%$ des extractions dentaires; ce risque de bactériémie lors d'extractions dentaires augmenterait avec l'âge des patients, l'inflammation périapicale et gingivale, la durée des soins (au delà de 100 minutes), le saignement (plus de $50 \mathrm{ml}$ ) et le nombre de dents extraites (on retrouve $100 \%$ de bactériémie à partir de 15 extractions dentaires pratiquées au cours d'une même séance). D'autres études présentent des chiffres similaires, avec un risque de bactériémie après extraction dentaire compris entre 51 et $100 \%$ [15]. Les bactériémies transitoires après soins dentaires sont détectables 5 minutes après le début des soins et persistent 10 à 30 minutes [16]. Les bactéries retrouvées lors de ces épisodes de bactériémie sont surtout des bactéries anaérobies [10, 14].

Une étude de Maestre et al. [17] en 2008 a montré que les surfaçages radiculaires provoquaient une bactériémie dans $77 \%$ des cas. Ici encore, les bactéries retrouvées étaient le plus souvent anaérobies (Prevotella spp., Micromonas micros et Fusobacterium nucleatum).

En 1986, Bender et Montgomery [18] ont montré que les traitements endodontiques non chirurgicaux ne produisent pas par eux-mêmes de bactériémies, mais que les manipulations associées pourraient être à l'origine de bactériémies. Celles-ci seraient largement évitées par des mesures simples d'éradication des micro-organismes buccaux à l'aide d'antiseptiques buccaux préopératoires. Ces auteurs ont analysé la bactériémie consécutive à différents soins dentaires, immédiatement après les soins et 10 minutes plus tard. Les résultats laissent penser que la fréquence de la bactériémie immédiate après les soins dentaires est dépendante du degré traumatique, de la concentration locale des bactéries et de l'importance de l'inflammation gingivale.

Il semble qu'il y ait beaucoup plus de bactériémies spontanément induites par les actes de la vie quotidienne que de bactériémies induites par des soins dentaires [19]. Il est en effet clairement établi que des bactériémies d'origine dentaire surviennent spontanément et quotidiennement en dehors de tout soin particulier [20]. La mastication de chewing-gum, par exemple, entraînerait une bactériémie dans 17 à 51 \% des cas [15, 21]. Pour Bhanji et al. [22], le brossage dentaire manuel entraîne des bactériémies dans $46 \%$ des cas et le brossage électrique dans $78 \%$ des cas. Selon ces auteurs, un brossage dentaire régulier pendant un mois expose à un risque cumulé de 5376 minutes de bactériémie, alors qu'une extraction dentaire non compliquée entraîne seulement 6 minutes de bactériémie en moyenne. De même, Roberts et al. [23] ont retrouvé une bactériémie dans 38,5\% des cas après un brossage dentaire, celle-ci ne durant que quelques secondes. A titre de comparaison, dans une autre étude, la même équipe a montré que le temps moyen de la bactériémie après extractions dentaires était d'environ 11 minutes [24]. 
Au total, il y a beaucoup plus de bactériémies induites par les actes de la vie quotidienne (brossage dentaire, mastication) que par les soins dentaires [25] (NP 2).

Bactériémies provoquées par les procédures bucco-dentaires [15].

\begin{tabular}{ll}
\hline Procédures & $\%$ de bactériémie \\
\hline $\begin{array}{l}\text { Extraction dentaire } \\
\text { simple }\end{array}$ & $40-50$ \\
$\quad$ multiple & $70-100$ \\
$\begin{array}{l}\text { Parodontologie } \\
\text { non chirurgicale } \\
\quad \text { détartrage surfaçage }\end{array}$ & $10-90$ \\
$\quad$ chirurgicale & \\
$\quad$ lambeau d'accès & $40-90$ \\
$\quad$ gingivectomie & 80 \\
Endodontie & \\
$\quad$ non chirurgicale & \\
$\quad$ intracanalaire & \\
$\quad$ extracanalaire & $0-30$ \\
$\quad$ chirurgicale & $0-50$ \\
$\quad$ curetage péri-apical & \\
$\quad$ élévation de lambeau & 30 \\
Anesthésie & 80 \\
$\quad$ péri-apicale & \\
intra-ligamentaire & 20 \\
Pose de bagues d'orthodontie & 10 \\
Pose de la digue & 30 \\
Pose de matrices & 30 \\
\hline
\end{tabular}

Les conséquences des bactériémies d’origine dentaire doivent par ailleurs être relativisées. Shariff et al. [26] en 2004 ont en effet évalué, dans une étude à la fois prospective et rétrospective, les relations entre les bactéries de la cavité buccale et les infections chez les patients hémodialysés. Aucune hémoculture réalisée chez 87 patients n'a permis de retrouver de micro-organismes buccaux.

\subsection{Classifications des foyers infectieux}

Parmi les situations qui correspondent à un risque infectieux, on peut distinguer :

- les FIBD (actifs ou latents): présence effective de foyers bactériens, qu'il s'agisse d'une infection avérée ou qu'il n'y ait pas de répercussion clinique au moment de l’observation ;

- les situations à risque infectieux potentiel (SRIP) : susceptibles de devenir des foyers infectieux dans le futur du fait des conditions réunies à l'échelon local. Cette éventualité ne peut être évaluée que d'un point de vue statistique.

Dans la littérature, aucune étude basée sur des arguments scientifiques ne propose de classification des situations à risque infectieux. Pour pallier ce manque et aider les lecteurs à se faire une idée de la 
virulence des situations les plus fréquemment rencontrées, un questionnaire a été soumis au groupe de cotation composé de chirurgiens dentistes et de stomatologues (G2).

Il s'agissait d'estimer le degré de virulence de diverses situations cliniques. Cette échelle reproduit la moyenne des avis du groupe de cotation sur la virulence présumée des différentes situations, en prenant pour base le cas d'une dent naturelle sur l'arcade et en estimant le risque supplémentaire, par rapport à cette dent saine, au moment de l'examen (sans présager de l'évolution éventuelle de cette virulence dans le temps): de 0 (pas de risque supplémentaire) à 10 (risque infectieux maximal). La gradation obtenue est exposée dans le tableau ci-dessous. Les valeurs représentent uniquement des avis d'experts : ce tableau n'a pas de valeur scientifique et il ne figure donc dans ce document qu'à titre indicatif. 
Virulence présumée dans diverses situations cliniques : modélisation graphique des avis d'experts du groupe G2

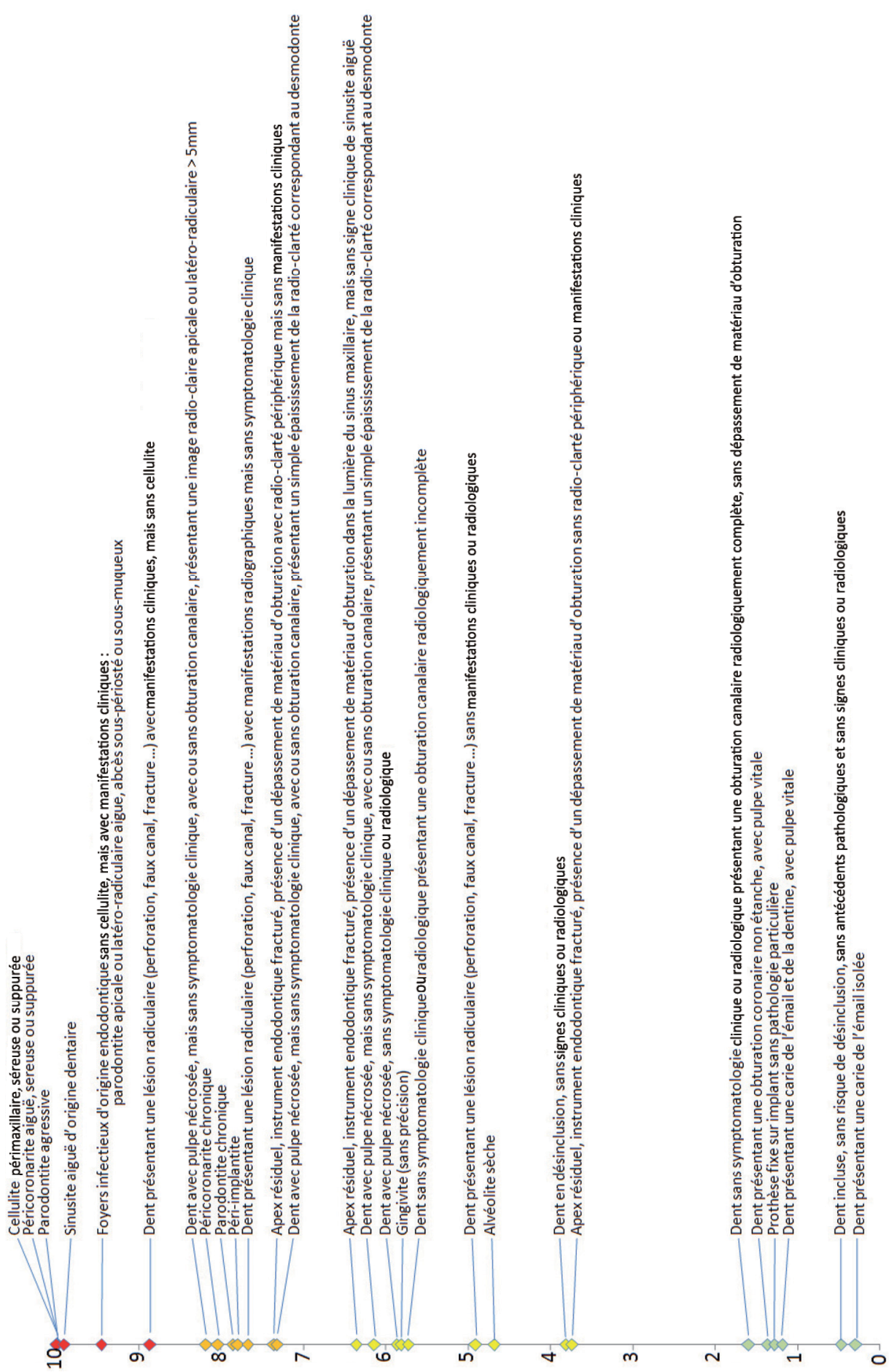

(risque infectieux supplémentaire par rapport à une dent saine sur l'arcade, de 0 à 10) 
Virulence présumée dans diverses situations cliniques:

tableau détaillé des avis d'experts du groupe $\mathrm{G} 2$

(risque infectieux supplémentaire par rapport à une dent saine sur l'arcade, de 0 à 10)

\begin{tabular}{|c|c|c|c|}
\hline & Situations & $\begin{array}{l}\text { Virulence } \\
\text { estimée }\end{array}$ & Ecart-type \\
\hline \multirow{6}{*}{ 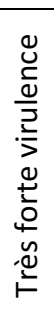 } & Cellulite périmaxillaire, séreuse ou suppurée & 10.00 & 0.00 \\
\hline & Péricoronarite aiguë, séreuse ou suppurée & 10.00 & 0.00 \\
\hline & Parodontite agressive & 10.00 & 0.00 \\
\hline & Sinusite aiguë d'origine dentaire & 9.91 & 0.20 \\
\hline & $\begin{array}{l}\text { Foyers infectieux d'origine endodontique sans cellulite, mais avec manifestations } \\
\text { cliniques : par odontite apicale ou latéro-radiculaire aiguë, abcès sous-périosté } \\
\text { ou sous-muqueux }\end{array}$ & 9.46 & 0.56 \\
\hline & $\begin{array}{l}\text { Dent présentant une lésion radiculaire (perforation, faux canal, fracture...) avec } \\
\text { manifestations cliniques, mais sans cellulite }\end{array}$ & 8.86 & 1.10 \\
\hline \multirow{7}{*}{ 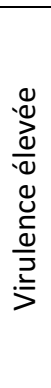 } & $\begin{array}{l}\text { Dent avec pulpe nécrosée, mais sans symptomatologie clinique, avec ou sans obturation } \\
\text { canalaire, présentant une image radio-claire apicale ou latéro-radiculaire }>5 \mathrm{~mm}\end{array}$ & 8.18 & 1.17 \\
\hline & Péricoronarite chronique & 8.05 & 1.06 \\
\hline & Parodontite chronique & 7.86 & 0.84 \\
\hline & Péri-implantite & 7.80 & 1.48 \\
\hline & $\begin{array}{l}\text { Dent présentant une lésion radiculaire (perforation, faux canal, fracture...) avec } \\
\text { manifestations radiographiques mais sans symptomatologie clinique }\end{array}$ & 7.68 & 1.23 \\
\hline & $\begin{array}{l}\text { Apex résiduel, instrument endodontique fracturé, présence d'un dépassement de } \\
\text { matériau d'obturation avec radio-clarté périphérique mais sans manifestations cliniques }\end{array}$ & 7.36 & 1.21 \\
\hline & $\begin{array}{l}\text { Dent avec pulpe nécrosée, mais sans symptomatologie clinique, avec ou sans obturation } \\
\text { canalaire, présentant une image radio-claire apicale ou latéro-radiculaire }<5 \mathrm{~mm}\end{array}$ & 7.32 & 1.35 \\
\hline \multirow{9}{*}{ 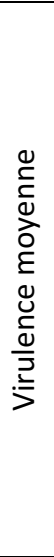 } & $\begin{array}{l}\text { Apex résiduel, instrument endodontique fracturé, présence d'un dépassement de } \\
\text { matériau d'obturation dans la lumière du sinus maxillaire, mais sans signe clinique de } \\
\text { sinusite aiguë }\end{array}$ & 6.36 & 1.91 \\
\hline & $\begin{array}{l}\text { Dent avec pulpe nécrosée, mais sans symptomatologie clinique, avec ou sans obturation } \\
\text { canalaire, présentant un simple épaississement de la radio-clarté correspondant au } \\
\text { desmodonte }\end{array}$ & 6.14 & 1.55 \\
\hline & Dent avec pulpe nécrosée, sans symptomatologie clinique ou radiologique & 5.86 & 1.67 \\
\hline & Gingivite (sans précision) & 5.82 & 1.66 \\
\hline & $\begin{array}{l}\text { Dent sans symptomatologie clinique ou radiologique présentant une obturation canalaire } \\
\text { radiologiquement incomplète }\end{array}$ & 5.73 & 1.74 \\
\hline & $\begin{array}{l}\text { Dent présentant une lésion radiculaire (perforation, faux canal, fracture...) sans } \\
\text { manifestations cliniques ou radiologiques }\end{array}$ & 4.91 & 1.38 \\
\hline & Alvéolite sèche & 4.68 & 2.03 \\
\hline & Dent en désinclusion, sans signes cliniques ou radiologiques & 3.82 & 1.08 \\
\hline & $\begin{array}{l}\text { Apex résiduel, instrument endodontique fracturé, présence d'un dépassement de } \\
\text { matériau d'obturation sans radio-clarté périphérique ou manifestations cliniques }\end{array}$ & 3.73 & 1.01 \\
\hline \multirow{6}{*}{ 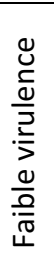 } & $\begin{array}{l}\text { Dent sans symptomatologie clinique ou radiologique présentant une obturation canalaire } \\
\text { radiologiquement complète, sans dépassement de matériau d'obturation }\end{array}$ & 1.59 & 1.07 \\
\hline & Dent présentant une obturation coronaire non étanche, avec pulpe vitale & 1.36 & 0.95 \\
\hline & Prothèse fixe sur implant sans pathologie particulière & 1.27 & 0.88 \\
\hline & Dent présentant une carie de l'émail et de la dentine, avec pulpe vitale & 1.18 & 0.90 \\
\hline & $\begin{array}{l}\text { Dent incluse, sans risque de désinclusion, sans antécédents pathologiques et sans signes } \\
\text { cliniques ou radiologiques }\end{array}$ & 0.46 & 0.46 \\
\hline & Dent présentant une carie de l'émail isolée & 0.28 & 0.46 \\
\hline
\end{tabular}


Un autre questionnaire a été adressé à ce même groupe de cotation G2, qui concernait la définition et la prise en charge des foyers infectieux, selon la méthode du consensus formalisé (Cf. méthologie). Les résultats obtenus ont permis de recueillir un consensus professionnel fort pour les situations suivantes :

- Une dent vitale atteinte d'une carie dont le traitement ne comporte pas de risque d'effraction pulpaire ne représente pas un foyer infectieux susceptible de dissémination (APF).

- Par comparaison avec le traitement endodontique d'une dent vitale,

- le traitement endodontique d'une dent nécrosée

- $\quad$ une reprise de traitement endodontique

représentent un risque majoré d'entraîner une bactériémie (APF).

- Une dent nécrosée représente un FIBD ou une SRIP (situation à risque infectieux potentiel) (APF).

- Les dents en désinclusion représentent des foyers infectieux actifs ou latents (APF).

En revanche, aucun consensus n'a pu être obtenu pour les situations suivantes :

- Pas de consensus pour affirmer que par comparaison avec une dent vitale, un implant dentaire représente un risque infectieux majoré (à noter que dans la gradation des situations à risque infectieux établie par le groupe de cotation, la virulence d'une prothèse unitaire sur implant est toutefois estimée légèrement supérieure à celle de la dent naturelle) (PC).

- Pas de consensus pour affirmer qu'une dent saine située dans un faisceau d'irradiation thérapeutique (ayant une probabilité supérieure d'atteinte carieuse du fait notamment de l'asialie) représente un risque infectieux majoré (PC).

\section{Situations cliniques dans lesquelles une recherche de FIBD doit être menée}

Il est du devoir du spécialiste de la cavité buccale de rechercher systématiquement les FIBD. La position privilégiée du médecin généraliste en fait également un acteur de santé à même de dépister les maladies infectieuses dentaires et parodontales lors d'un examen clinique systématique.

Les infections buccales étant susceptibles d'interagir avec l'état général, il est particulièrement nécessaire de rechercher la présence de FIBD dans un certain nombre de situations cliniques.

\subsection{Patients présentant un sepsis et/ou un syndrome inflammatoire chronique à distance de la cavité buccale}

\subsubsection{Sepsis et infections focales}

Le sepsis est défini par l'association d'une réaction inflammatoire généralisée et d'une défaillance d'organe, secondaire à une infection. Un sepsis sévère peut conduire à un choc septique défini comme une hypotension induite, persistant malgré un remplissage vasculaire adapté [27]. L'agent pathogène causal est le plus souvent une bactérie (plus rarement il peut s'agir d'un virus, d'un champignon ou 
d'un parasite). Le point d'appel infectieux retrouvé reste plus fréquemment urinaire, abdominal, cutané et surtout pulmonaire actuellement.

L'orientation vers une recherche de FIBD se fera selon le type de bactérie retrouvée.

Dans la littérature, de nombreux travaux mettent en exergue la gravité et les conséquences des infections à distance d'origine bucco-dentaire. Une étude rétrospective [28], portant sur l'observation de 35 patients ayant nécessité une hospitalisation, rapporte un taux de complications générales (septicémies, endocardites, abcès cérébraux...) de $29 \%$. II y a eu trois décès parmi les patients ayant eu une complication générale ; les trois avaient une pathologie générale grave associée.

L'incidence générale des abcès cérébraux est d'environ 1/100 000 . Ils peuvent avoir différentes étiologies: post-traumatique, diffusion par contiguïté, origine hématogène métastatique. L'abcès cérébral d'origine hématogène se manifeste surtout dans les territoires frontaux, pariétaux ou frontopariétaux [29]. Brewer et al. [30] ont répertorié 60 cas d'abcès cérébraux, parmi lesquels une origine dentaire a été mise en évidence dans 6,6\%. Dans une étude rétrospective, sur 163 patients hospitalisés pour un abcès du cerveau, Roche et al. [31] ont trouvé une origine dentaire dans moins de $2,5 \%$ des cas ; l'origine sinusienne était la plus fréquente (34\%).

La flore microbienne présente dans les abcès cérébraux d'origine dentaire est polymorphe. On retrouve dans $70 \%$ des cas des streptocoques, ainsi que des Bacteroides et Fusobacterium. Une méningite accompagne parfois l'abcès cérébral et peut être le premier signe clinique décelé. Dans 0,3 à 2,4\% des cas, les streptocoques oraux sont les micro-organismes incriminés [32].

Les abcès du foie à pyogènes sont le plus souvent d'origine digestive, l'infection se faisant par voie biliaire ou portale. Seuls quelques cas d'origine dentaire ont été rapportés dans la littérature. ॥ s'agissait le plus souvent d'infection à Fusobacterium nucleatum [33, 34].

Des travaux ont également établi une relation entre des pneumonies et des parodontites [35]. Le risque de développer une pneumonie apparait 1,67 fois supérieur si les patients n'ont pas bénéficié de soins dentaires actifs; à l'inverse, le traitement des parodontites permettrait de réduire ce risque [36].

\subsubsection{Syndrome inflammatoire chronique}

Les fièvres (fièvre, fièvre chronique, fièvre ondulante) sont le plus souvent révélatrices d'un processus bactérien ou viral. L'examen clinique est très important et doit tenir compte d'une possible pathologie bucco-dentaire. Cependant, l'étude de la littérature n'a permis de retrouver que quelques rapports de cas où cette association a pu être établie.

\subsubsection{Pathologies immunes déclenchées ou favorisées par une infection}

Il est évoqué qu'une pathologie immune puisse être déclenchée par une infection. Là encore, on ne retrouve que quelques cas rapportés mettant en cause des infections bucco-dentaires [37].

Les patients atteints de polyarthrite rhumatoïde souffrent plus tôt, plus souvent et de formes plus sévères de parodontites, que le reste de la population [38]. Plusieurs études [39, 40] ont suggéré que le traitement de la maladie parodontale réduit l'activité de la polyarthrite rhumatoïde (NP 2). 


\subsection{Patients présentant un risque majoré d'infection bucco-dentaire du fait d'une radiothérapie cervico-faciale}

La radiothérapie cervico-faciale expose au risque d'ostéonécrose.

Une radiothérapie externe est réalisée dans plus de $70 \%$ des cancers des voies aérodigestives supérieures. Les complications bucco-dentaires d'une radiothérapie cervico-faciale découlent d'effets secondaires qui touchent la muqueuse buccale, les os maxillaire et mandibulaire, les glandes salivaires ou les dents [41].

Les caries dentaires surviennent spontanément dans les quatre à six mois qui suivent la fin de la radiothérapie externe $[42,43]$. Elles évoluent en des sites peu habituels et n'épargnent aucune dent. Elles atteignent essentiellement les collets, sous la forme de caries rampantes annulaires qui évoluent jusqu'à la fracture corono-radiculaire, mais aussi, après érosion, les bords libres et les pointes occlusales. Ces caries post-radiques se distinguent des caries banales par leur plus grande agressivité et leur rapidité d'évolution. Elles sont liées à l'hyposialie et à l'acidité buccale. Sans traitement, elles peuvent aboutir à une ostéoradionécrose. Cette complication majeure est également susceptible d'apparaître à la suite d'avulsions dentaires ou d'effractions muqueuses (notamment ulcérations par prothèse amovible), voire aussi sans cause apparente.

Classiquement, l'ostéoradionécrose survient de quelques semaines à plusieurs années après une irradiation (81 \% dans les trois ans) [44]. Dans une série de 413 patients ayant bénéficié d'une radiothérapie cervico-faciale, 8,9\% ont développé une ostéoradionécrose [44].

Al-Nawaz et Grötz [45] ont étudié le changement de la flore buccale chez 22 patients après une irradiation supérieure à $30 \mathrm{~Gy}$. Cinq micro-organismes pathogènes parodontaux ont été étudiés (Aggregatibacter actinomycetemcomitans, Porphyromonas gingivalis, Prevotella intermedia, Tannerella forsythensis, Treponema denticola) à l'aide de sondes ADN, ainsi que la présence de lactobacilles et de Streptococcus mutans. Aucun changement n'a été observé pour les pathogènes parodontaux en contraste avec une augmentation des pathogènes impliqués dans le développement des caries.

Les hyposialies, quelle que soit leur origine, majorent le risque d'infection du fait des modifications qualitatives et quantitatives de la salive. Des études [42, 46, 47] permettent de mettre en évidence une dose de tolérance de la glande parotide située autour de 25 à 30 Gy en moyenne (NP 2). Cependant, même si la dose délivrée est faible, il existe une période post-thérapeutique de diminution importante du flux salivaire. La vitesse de récupération est inversement proportionnelle à la dose reçue et la période de récupération s'étale sur un à deux ans.

Dans la mesure où l'épargne d'une ou de deux glandes submandibulaires ne compromet pas le contrôle tumoral, la dose moyenne délivrée au tissu glandulaire ne devrait pas excéder 39 Gy [48]. Le bénéfice clinique de l'épargne des glandes submandibulaires n'est cependant pas démontré actuellement.

Irradiation et implants dentaires

Les rares publications sur ce sujet sont presque toujours des études rétrospectives à faible effectif [49]. Les échecs implantaires sont relativement rares mais imprévisibles. Ils surviennent exclusivement pour 
une irradiation supérieure à 45 Gy. II s'agit le plus souvent d'absence ou de perte d'ostéointégration et exceptionnellement d'ostéoradionécrose.

Ben Slama et al. [50] ont rapporté un cas d’ostéoradionécrose développée 3 mois après la fin de la radiothérapie autour d'implants ostéointégrés depuis 10 ans.

Ozen et al. [51] ont rapporté, dans une étude in vitro, un accroissement de $21 \%$ de la dose d'irradiation au voisinage des implants en titane (jusqu'à $2 \mathrm{~mm}$ ). Cette constatation suggère un facteur de risque supplémentaire autour des implants.

Recommandation proposée : Avant toute radiothérapie cervico-faciale, un bilan bucco-dentaire doit être entrepris au plus tôt (APF).

\subsection{Patients susceptibles de développer une infection à point de départ bucco-dentaire du fait de facteurs généraux}

\subsubsection{Situations en relation avec un état pathologique}

\subsubsection{Infection VIH}

Des gingivites et des parodontites ulcéro-nécrotiques sont rapportées dans 16 à $17 \%$ des patients infectés par le virus d'immunodéficience humaine (VIH) en Afrique [52] et $23 \%$ en Inde. En Europe, ce taux oscillait entre 10 et $19 \%$ avant l'avènement des trithérapies antirétrovirales [53, 54].

Parmi les lésions orales fortement corrélées au VIH, on retrouve les gingivites et les parodontites ulcéro-nécrotiques. Rams et al. [55] ont évalué le statut sérologique de patients présentant une gingivite ulcéro-nécrotique (GUN). Les résultats indiquaient que $69 \%$ des patients étaient séropositifs et que cette lésion était souvent la première manifestation de la maladie. De plus, il existe une corrélation entre la diminution du nombre de CD4 et la probabilité de développer une GUN : les patients présentant un taux de CD4<200/mm3 ont 20,8 fois plus de risque de développer une GUN que les patients ayant un taux de CD4>200/mm3.

Recommandation proposée: Lors de la découverte d'une séropositivité VIH, il est souhaitable de réaliser un bilan bucco-dentaire à la recherche de FIBD (APF).

\subsubsection{Insuffisance rénale}

Un état bucco-dentaire défectueux est très souvent constaté chez les patients hémodialysés, qui s'explique notamment par la fréquence d'une mauvaise hygiène bucco-dentaire. Cependant, la littérature est discordante. Bayraktar et al. [56] ont montré dans une étude cas-témoin que les patients dialysés présentaient davantage de plaque dentaire, de tartre et de saignements gingivaux. Cependant les profondeurs de sondage n'étaient pas statistiquement significatives (NP 3). L'étude castémoin de Garcez et al. [57] portant sur des patients non dialysés, n’a pas mis en évidence de différence significative entre les patients insuffisants rénaux et le groupe contrôle (NP 3). 
Shariff et al. [26] ont étudié les infections sur les voies veineuses chez les patients dialysés. Ils ont conclu que les bactéries d'origine buccale sont rarement, voire jamais en cause dans ce type d'infection.

Dans une étude transversale portant sur 11211 adultes, Fischer et al. [58] ont estimé que la parodontite pourrait être un facteur de risque de l'insuffisance rénale chronique au même titre que I'hypertension, le tabac et l'hypercholestérolémie.

\subsubsection{Diabète sucré}

La relation entre diabète sucré et maladies parodontales a été établie par de nombreuses études épidémiologiques, parfois contradictoires. Celles-ci s'accordent toutefois, dans leur grande majorité, à démontrer d'une part que le diabète constitue un facteur de risque susceptible de favoriser le développement d'une parodontite, et d'autre part que l'infection parodontale semble perturber l'équilibre glycémique.

Grossi et al. [59] ont suivi 113 sujets souffrant d'un diabète de type II et d'une parodontite; ils les ont répartis en 5 groupes selon le traitement parodontal reçu au cours de la préparation initiale. Les groupes traités par $100 \mathrm{mg}$ de doxycycline par voie générale pendant deux semaines avaient un taux d'hémoglobine glyquée $(\mathrm{HbA} 1 \mathrm{c})$ réduit de $10 \%$ par rapport à la valeur initiale ; cependant ce taux remontait légèrement entre 3 et 6 mois et, pour les auteurs, cette situation pourrait correspondre au retour d'une flore pathogène qui n'aurait pas été totalement éradiquée.

Christgau et al. [60] n'ont pas constaté d'amélioration significative du contrôle métabolique du diabète (insulino ou non insulino-dépendant) après mise en état parodontal. Cependant leur groupe de patients avait un bon contrôle métabolique du diabète dès le départ.

Pour Taylor [61], le traitement parodontal permet un meilleur contrôle de la glycémie. Une métaanalyse confirme ces résultats [62].

L'infection parodontale apparaît de plus en plus comme un facteur potentiel de perturbation des équilibres homéostasiques, susceptible d'entraîner des manifestations pathologiques à distance du foyer d'origine.

Au total, l'éradication des FIBD améliore le contrôle de la glycémie chez les patients diabétiques (NP 1) et le traitement parodontal améliore le contrôle de la glycémie dans les trois mois (NP 2).

Recommandation proposée : Dès le diagnostic d'un diabète, il est recommandé de pratiquer un bilan bucco-dentaire (APF).

\subsubsection{Athérosclérose}

Janket et al. [63] ont observé dans une méta-analyse que les maladies parodontales étaient associées à une augmentation de $19 \%$ du risque de développement de maladies cardio-vasculaires (NP 1).

Au cours des 15 dernières années, de nombreuses études épidémiologiques ont montré qu'il existait une relation entre l'infection parodontale et les maladies coronariennes, avec cependant de grandes variations dans la force de ce lien. Une méta-analyse de 2008 [64] montre que la maladie parodontale 
constitue un facteur de risque indépendant des autres risques connus des coronaropathies, et dont le risque relatif est estimé entre 1,24 et 1,35 (NP 1).

Selon une autre méta-analyse [65], la prévalence et l'incidence des coronaropathies sont significativement augmentées chez les patients atteints de parodontites.

Une autre étude [66] a montré que la maladie parodontale était également un facteur de risque des accidents vasculaires cérébraux, particulièrement les accidents vasculaires cérébraux non hémorragiques.

Les mécanismes de cette association ne sont pas encore clairement établis. Parmi les liens directs, on peut évoquer l'inflammation générale, révélée par l'augmentation de différents biomarqueurs dont la CRP [67]. Parmi les autres hypothèses avancées, il y a la possibilité que certains micro-organismes parodontaux puissent initier ou exacerber un processus pathologique sur la paroi des artères du fait de leur activité pro-agrégantes (théorie du thrombus infecté). Concernant les liens indirects, les parodontopathies et l'athérosclérose partageraient les mêmes facteurs de risque comme le tabac, l'obésité, l'hyperlipidémie, le diabète.

\subsubsection{Infections broncho-pulmonaires}

La pneumonie est une infection du parenchyme pulmonaire causée par une grande variété d'agents infectieux. Elle peut mettre la vie en danger, en particulier chez le patient âgé ou immunodéprimé. Les bactéries anaérobies peuvent être à l'origine de pneumonies, et la plaque dentaire apparaît logiquement comme une origine de ces bactéries, notamment chez les patients atteints de maladies parodontales [68-70].

\subsubsection{Affections dermatologiques}

La recherche bibliographique n'a pas permis de retrouver de série d'infections bucco-dentaires en rapport avec une affection dermatologique. Des cas cliniques isolés ont cependant été rapportés. Lesclous et Maman [71] ont par exemple observé la disparition d'une rosacée à la suite du traitement d'une infection bucco-dentaire.

En l'absence de données avérées, il est légitime d'éliminer les causes éventuelles d'irritation buccale. II convient ainsi de rechercher et de traiter les FIBD devant un lichen buccal pour éviter une aggravation par un phénomène de Koebner. De même, devant un érysipèle de la face, on peut rechercher un FIBD ou un foyer sinusien [72].

\subsubsection{Autres situations sans niveau de preuve}

Dans un certain nombre de situations pathologiques, une cause bucco-dentaire a pu être évoquée, sans que la littérature scientifique puisse en apporter la preuve formelle. C'est le cas notamment de l'uvéite, de la pelade.

Aujourd'hui, un consensus se dégage en dermatologie pour considérer que les cas qui ont été rapportés n'étaient probablement que des coïncidences et qu'il n'y a pas lieu de rechercher une origine dentaire ou parodontale devant un cas de pelade. 
De même, en ophtalmologie, il semble que les progrès de l'immunologie des 30 dernières années ont clarifié les étiologies des uvéites. Il est aujourd'hui généralement admis qu'il n'y a pas lieu de rechercher une origine dentaire ou parodontale devant un cas d'uvéite.

En matière de médecine sportive, il n'y a pas non plus de preuve pour affirmer qu'un FIBD peut être à l'origine d'une tendinite.

\subsubsection{Situations liées à des prises médicamenteuses}

\subsubsection{Traitements immunosuppresseurs}

Dans le cadre des transplantations et de maladies auto-immunes, on retrouve les préconisations suivantes :

Little et Rhodus [73] suggèrent, avant une transplantation hépatique, une évaluation bucco-dentaire avec l'élimination des foyers actifs. Pour les patients présentant un bon état bucco-dentaire, ils suggèrent que le traitement soit le plus conservateur possible, avec le renforcement des mesures de prévention. En revanche, ils conseillent de traiter les patients ayant une mauvaise hygiène buccodentaire et présentant des atteintes parodontales sévères ou de nombreuses caries avec ou sans foyers périapicaux, par des extractions suivies d'une réhabilitation prothétique.

Little et al. [74] suggèrent également qu'avant chimiothérapie ou greffe de cellules souches hématopoïétiques, les soins dentaires et/ou parodontaux soient accomplis aussitôt que possible avant la greffe et au moins une semaine avant le début de la chimiothérapie.

Outre les greffes et transplantations d'organes, plusieurs maladies auto-immunes sont susceptibles d'être traitées par des traitements immunosuppresseurs ou immunomodulateurs. Parmi les plus fréquentes:

- polyarthrite rhumatoïde

- lupus érythémateux systémique

- purpura thrombopénique immunologique

- anémie hémolytique auto-immune

- érythroblastopénie auto-immune

- polymyosites et dermatomyosites

- syndrome de Sjögren

- cryoglobulinémies

- vascularites

- auto-anticorps anti-facteur VIII

- micro-angiopathies thrombotiques

- glomérulonéphrite extramembraneuse

- pemphigus vulgaire

- myasthénie

- neuropathies avec anticorps anti-MAG

- sclérose en plaques

Ces maladies auto-immunes peuvent être traitées par différents traitements immunomodulateurs : 


\subsection{Traitements par corticoïdes}

L'inhibition du système immunitaire et du processus inflammatoire représentent deux éléments centraux de l'action des glucocorticoïdes. Il en découle une augmentation du risque infectieux pendant la corticothérapie. De plus, leur action anti-inflammatoire masque en partie les signes de l'infection, ce qui peut en retarder le diagnostic et donc la prise en charge.

Dans une étude rétrospective, analysant 71 études cliniques, Stuck et al. [75] ont évalué l'augmentation du risque relatif d'infection à 1,6 chez les patients sous corticothérapie au long cours. Le taux d'infection n'était pas augmenté pour les patients recevant une dose inférieure à $10 \mathrm{mg}$ équivalent prednisone par jour. Bien que ce risque ne soit pas fortement augmenté par des doses modérées de glucocorticoïdes, les infections opportunistes et les complications infectieuses semblent néanmoins plus nombreuses en dehors des cures courtes.

Recommandation proposée : Une corticothérapie par voie générale ne justifie pas une prise en charge particulière du fait de sa seule présence :

- si elle a une posologie inférieure à $10 \mathrm{mg} / \mathrm{j}$ équivalent prednisone (APF) ou

- si elle a une durée inférieure à 8 jours pour une posologie inférieure ou égale à $1 \mathrm{mg} / \mathrm{Kg} / \mathrm{j}$ équivalent prednisone (APF).

\subsection{Thérapeutiques biologiques ciblées à visée immunosuppressive}

Les thérapeutiques ciblées à visée immunosuppressive issues du génie biologique sont aujourd'hui largement utilisées dans de nombreuses spécialités (rhumatologie, cancérologie, hématologie, immunologie, dermatologie, gastro-entérologie ...). II s'agit par exemple de :

- abatacept $\quad\left(\right.$ Orencia $\left.^{\circledR}\right)$

- adalimumab $\left(\right.$ Humira $\left.^{\circledR}\right)$

- alemtuzumab (Mabcampath $\left.{ }^{\circledR}\right)$

- etanercept $\left(\right.$ Enbrel $\left.^{\circledR}\right)$

- infliximab (Rémicade ${ }^{\circledR}$ )

- ofatumumab (Arzerra $\left.{ }^{\circledR}\right)$

- rituximab (Mabthera $\left.{ }^{\circledR}\right)$

- tocilizumab (RoActemra $\left.{ }^{\circledR}\right)$

Leurs liens avec les infections bucco-dentaires résident sur leur aptitude à inhiber une ou plusieurs voies de la réponse immune adaptative. Bien que leurs mécanismes d'action soient différents, il existe un dénominateur commun aboutissant à fragiliser les défenses immunitaires et favoriser les infections. L'infliximab, l'adalimumab et l'etanercept inhibent le TNF alpha, l'abatacept les voies de costimulation, le rituximab et l'ofatumumab entraînent une lymphopénie $B$, l'alemtuzumab une lymphopénie sévère $B$ et $T$, et le tocilizumab inhibe l'interleukine 6. 
La prise de ces nouveaux immunosuppresseurs représente un important facteur de risque pour les infections bucco-dentaires. La conduite à tenir chez les patients traités est détaillée au paragraphe 4.1.2. (p 41)

\subsection{Autres immunosuppresseurs}

La ciclosporine, le tacrolimus, le sirolimus et l'everolimus font partie, du fait de leur activité immunosuppressive, des thérapeutiques destinées à réduire le risque de rejet après transplantation d'un organe solide ou de maladie du greffon contre l'hôte après greffe de cellules souches hématopoïétiques. Les patients greffés d'organe ou de cellules souches hématopoïétiques sont souvent traités par des associations d'immunosuppresseurs incluant habituellement des corticoïdes. II convient donc de les considérer comme à risque infectieux particulièrement élevé.

NB : Les chimiothérapies anticancéreuses, potentiellement immunosuppressives, sont évoquées au chapitre suivant pour plus de lisibilité.

Recommandation proposée: Un bilan bucco-dentaire doit être effectué au plus tôt avant une transplantation ou l'instauration d'un traitement immunosuppresseur dans la mesure où l'urgence à instaurer le traitement l'y autorise (APF).

\subsubsection{Chimiothérapie anticancéreuse}

Durant la chimiothérapie anticancéreuse, les infections bucco-dentaires ont une morbidité importante et peuvent parfois même entraîner le décès du patient [76]. Les patients présentent en effet un risque majoré d'infection, soit par l'apparition de nouvelles infections bucco-dentaires, soit par l'exacerbation de lésions chroniques [77, 78]. La chimiothérapie peut notamment induire des mucites, celles-ci pouvant constituer une porte d'entrée pour les bactéries et provoquer une bactériémie.

Il est à noter que les réponses inflammatoires sont souvent modifiées pendant la phase myélosuppressive: les signes habituels comme l'érythème et la tuméfaction peuvent être altérés; leur absence n'est pas donc suffisante pour exclure une infection [79].

Bergmann et al. [80] ont étudié les changements de la flore buccale et de la salive chez 10 patients suivis pour une leucémie. Ils ont observé une diminution de $64 \%$ du flux salivaire dans les 28 jours qui suivent le début de la chimiothérapie. Parallèlement ils ont noté un doublement du nombre de bactéries, sans changement qualitatif de la flore.

L'étude de Dreizen et al. [81] portant sur 1500 sujets atteints de leucémie a rapporté qu'un tiers de ces patients souffraient d'infections buccales pendant la chimiothérapie. Dans une autre étude [82], on retrouve un taux de 9,7\% d'infections buccales dans le cadre de différents types de cancers.

Les patients bénéficiant d'une chimiothérapie sont particulièrement sensibles aux infections parodontales lors des périodes de neutropénie [83]. 


\subsubsection{Bisphosphonates}

Recommandation proposée : À l'instauration d'un traitement par bisphosphonates (quelle qu'en soit l'indication), il est recommandé de pratiquer un bilan bucco-dentaire (CR).

Se référer aux recommandations de l'AFSSAPS*. Voir aussi paragraphe 4.4 (p. 45).

\subsection{Patients susceptibles de développer une infection focale à point de départ bucco-dentaire}

Les FIBD, même minimes, peuvent avoir à distance des répercussions importantes sur les différents appareils de l'organisme.

\subsubsection{Risque d'endocardite infectieuse}

L'exemple le mieux connu de surinfection liée à une localisation secondaire de la bactérie au décours d'un acte bucco-dentaire, est celui de l'endocardite infectieuse (EI).

L'El résulte de la colonisation, par des bactéries circulantes (bactériémie), d'une végétation fibrinoplaquettaire développée sur un endocarde le plus souvent lésé ou une sur prothèse valvulaire. Cette pathologie reste assez rare mais son incidence ne semble pas diminuer au cours des 10 dernières années malgré les efforts de prévention. Cette apparente stabilité masque de profonds changements épidémiologiques, avec une population atteinte de plus en plus âgée, l'augmentation de fréquence des endocardites sur prothèse et des endocardites à staphylocoques.

Dans l'étude épidémiologique prospective de Hoen et al. [84], conduite sur une année (1999) dans l'ensemble des hôpitaux de 6 régions représentant $26 \%$ de la population française (390 adultes atteints d'une El selon les critères de la Duke University), l'incidence de l'El en France métropolitaine, a été estimée à 31 nouveaux cas par million d'habitants et par an. L'incidence semble plus importante chez les hommes, avec 44 nouveaux cas par million d'habitants et par an contre 17 chez les femmes. L'évolution du profil microbiologique est marquée par l'augmentation de la fréquence des El à streptocoques du groupe D et à staphylocoques. Les auteurs ont observé une nette diminution de fréquence des El à streptocoques oraux qui sont passées de $27 \%$ en 1991 à 17 en 1999, avec parallèlement une augmentation de la fréquence des El à staphylocoque doré.

La plupart des séries ne rapportent qu'un nombre très faible d'El compliquant une procédure invasive bucco-dentaire (2,7 à $7 \%$ des séries épidémiologiques). Cependant, même en cas de concordance temporelle rétrospectivement évoquée entre la réalisation d'une procédure bucco-dentaire et la survenue d'une El, il n'est pas possible de déterminer avec certitude si la bactériémie à l'origine de l'EI a été provoquée par la procédure, si elle a été causée par la pathologie bucco-dentaire ayant motivé la

* Recommandations sur la prise en charge bucco-dentaire des patients traités par bisphosphonates (19/12/2007), http://www.afssaps.fr/Infos-de-securite/Lettres-aux-professionnels-de-sante/Recommandations-sur-la-prise-en-chargebucco-dentaire-des-patients-traites-par-bisphosphonates (consulté le 12/09/2009) 
réalisation de la procédure ou si elle a été induite par un acte de la vie quotidienne (brossage dentaire, mastication).

L'étude de Van der Meer et al. [85] conclut que les gestes dentaires ne causent que peu d'El, et la prophylaxie ne préviendrait qu'un faible nombre de cas. De la même façon, dans l'étude multicentrique de Strom et al. [86], les auteurs, confirment que les gestes dentaires ne sont pas un facteur de risque d'El, y compris chez les patients atteints d'une valvulopathie. Enfin, d'après Duval et al. [87], «il faut un nombre très important de doses de prophylaxie pour ne prévenir qu'un très petit nombre d’El».

De ce fait, la nécessité et l'efficacité d'une antibioprophylaxie systématique avant les gestes dentaires chez les sujets à risque ont été remises en question. Lors des dernières mises à jour, les recommandations des différentes sociétés savantes ont évolué de façon concordante vers la diminution des indications d'antibioprophylaxie et l'accent est mis sur la prévention par l'hygiène bucco-dentaire et la surveillance. Le groupe de cotation conseille de se reporter aux dernières recommandations en vigueur [88].

Actuellement, il est considéré que seuls les patients ayant un risque élevé d’El doivent recevoir une antibioprophylaxie [88].

\begin{tabular}{|l|}
\hline \multicolumn{1}{|c|}{ Situations à haut risque d'endocardite infectieuse } \\
\hline Prothèse valvulaire cardiaque \\
\hline Antécédent d'endocardite \\
\hline Cardiopathie congénitale : \\
- cyanogène non réparée, y compris shunts et conduits palliatifs \\
- complètement réparée avec matériel prothétique, placé par cathétérisme ou \\
- chirurgicalement, pendant les 6 mois suivant la procédure \\
réparée avec défauts résiduels sur le site ou adjacent au site du patch prothétique \\
\hline
\end{tabular}

NB : Voir aussi les recommandations du chapitre 3.1 sur la conduite de l'examen clinique (p. 34).

Les patients présentant des valvulopathies non opérées ont un risque modéré d'El et ne justifient pas une antibioprophylaxie [88].

Les autres situations, notamment les stimulateurs cardiaques implantés, les pontages coronaires et les endoprothèses vasculaires (stents) n'accroissent pas le risque d'El à point de départ bucco-dentaire [88].

\subsubsection{Risque lié à une prothèse articulaire}

Les infections de prothèses articulaires à point de départ bucco-dentaire sont rares et ne surviendraient que dans 0,04 à 0,2 \% du total des arthroplasties [89-93].

Une revue de la littérature [94] a rapporté un certain nombre de cas d'infections articulaires associées de manière forte à des foyers infectieux ou à des soins d'origine buccale. Toutefois, le niveau de 
preuve de la relation de causalité entre un FIBD et une infection prothétique reste faible. Il en est de même en ce qui concerne l'association entre un soin de la sphère buccale et une infection articulaire. Un risque élevé de bactériémie ne semble pas corrélé à un taux élevé d'infection sur arthroplastie. D'après cette revue, le staphylocoque est responsable de plus de $25 \%$ des infections de prothèse articulaire après soins dentaires, alors qu'il ne représente que 0,005 \% de la flore buccale et n'est que très rarement retrouvé lors des bactériémies d'origine dentaire.

Recommandation proposée : Toutefois, avant la mise en place d'une prothèse articulaire, il est recommandé d'effectuer un bilan bucco-dentaire au plus tôt (APF).

\subsection{Cas particulier de la grossesse}

Deux situations épidémiologiques ont été identifiées comme la première cause de morbidité et de mortalité néonatale [95] : il s'agit des accouchements prématurés (<37 semaines, avec un taux général en Europe de 5 à $9 \%$ ) et/ou du faible poids de naissance $(<2500 \mathrm{~g}$, soit $6,4 \%$ des naissances en Europe).

Plusieurs facteurs favorisants ont été mis en évidence dans ces phénomènes (niveau socioéconomique, âge au moment de la grossesse). On retrouve aussi les inflammations et les infections uro-génitales. Cependant, 50 \% des causes d'accouchement prématuré demeurent inconnues [96].

Les maladies parodontales ont été évoquées comme une possible source d'inflammation et d'infection en lien avec ce phénomène.

Certaines études ont en effet montré la présence de pathogènes parodontaux ( $P$ gingivalis) dans le placenta (NP 3) [97-99].

Par ailleurs, une étude de Rakoto-Alson et al.[100] menée chez 204 patientes malgaches enceintes a montré que l'indice de plaque et l'indice de saignement papillaire étaient significativement plus importants en cas d'accouchement prématuré ou de naissance de faible poids; de plus, la présence d'une parodontite était significativement associée au risque de prématurité (77 \% vs. 8 \%).

Une étude multicentrique [101] cas-témoin, menée chez 1108 femmes ayant eu un accouchement prématuré et 1094 femmes ayant accouché à terme, a montré un accroissement du risque de prééclampsie (odds ratio de 2,46 ) chez les patients ayant une parodontite généralisée. L'association entre parodontite et pré-éclampsie pourrait être liée à l'augmentation du taux de protéine $C$ réactive et d'autres médiateurs de l'inflammation (cytokines, PGE2) [102-104]. La parodontite serait ainsi un marqueur de susceptibilité à l'inflammation. Toutefois, le traitement de cette parodontite ne permettrait pas de réduire le risque d'accouchement prématuré [105].

La revue de la littérature rapporte des résultats contrastés quant à l'effet du traitement parodontal pendant la grossesse; il ne permettrait pas une diminution des naissances prématurées ou des insuffisances pondérales du nouveau né (NP 2). Le traitement parodontal est néanmoins réalisable pendant la grossesse, et de préférence au 2ème trimestre.

Recommandation proposée: Chez les femmes enceintes ou ayant un projet de grossesse, il est recommandé de pratiquer un bilan bucco-dentaire (APF). 


\section{Comment conduire la recherche des FIBD?}

Lorsqu'il s'agit de rechercher des FIBD dans un contexte médical, le bilan bucco-dentaire doit être particulièrement complet et minutieux. Il a pour objectifs de mettre en évidence l'ensemble des FIBD, patents ou latents et de révéler d'éventuels facteurs favorisant une infection. II nécessite un interrogatoire rigoureux ainsi que des examens cliniques et radiographiques, voire biologiques.

\subsection{Examen clinique}

\section{Interrogatoire}

L'interrogatoire s'attache d'abord à explorer les antécédents généraux et loco-régionaux du patient. II a pour but de définir l'état général du patient et donc les risques qui y sont inhérents. II faut aussi rechercher les signes généraux et fonctionnels évocateurs d'un FIBD.

\section{Examen physique}

Examen exo-buccal

Il doit rechercher l'ensemble des signes cliniques susceptibles d'être associés à une infection d'origine bucco-dentaire :

- tuméfaction cervico-faciale

- fistule cutanée

- adénopathies cervico-faciales, en précisant leurs caractéristiques (localisation, nombre, taille, consistance, adhérence, caractère inflammatoire)

Examen endo-buccal

L'examen de la denture, réalisé sous bon éclairage, à l'aide d'un miroir, d'une sonde droite, d'une sonde en crochet et d'une sonde parodontale, précisera :

- l'état dentaire général

- le nombre, la valeur intrinsèque et extrinsèque des dents restantes

- la présence de caries, l'existence de pertes de substance coronaire

- l'existence de fêlures ou de fractures

- la présence de dents en désinclusion

- l'existence et la qualité des restaurations coronaires

- les mobilités dentaires suivant l'indice de Mülheman

- 0 : ankylose

- 1 : mobilité physiologique perceptible entre deux doigts

- 2 : mobilité transversale visible à l'œil nu inférieure à $1 \mathrm{~mm}$

- 3 : mobilité transversale supérieure à $1 \mathrm{~mm}$

- 4 : mobilité axiale

- La vitalité pulpaire (utilisation de tests électriques ou thermiques). 
Dans leur étude évaluant les performances diagnostiques des différents tests, Petersson et al. [106] ont observé que pour des dents vivantes, la probabilité d'obtenir une sensibilité aux tests était évaluée à $90 \%$ pour le test au froid, $83 \%$ pour le test au chaud et $84 \%$ pour le test électrique; pour les dents nécrosées, la probabilité de ne pas obtenir une sensibilité aux mêmes tests était respectivement évaluée à $89 \%, 48 \%$ et $88 \%$. Les auteurs ont conclu qu'une réponse positive au froid et au test électrique était fortement associée à une probabilité de vitalité pulpaire.

- La modification de la teinte de la structure coronaire

- La présence de douleurs à la palpation ou à la percussion.

Examen du parodonte

Il est important de diagnostiquer une halitose éventuelle et d'en déterminer l'origine. Les composés sulfurés volatils produits par les bactéries à Gram négatif anaérobies, majoritairement parodontopathogènes, ont des effets toxiques sur les tissus parodontaux en fragilisant les muqueuses non kératinisées, en modifiant le squelette des fibroblastes, en activant les monocytes et en perturbant le processus de cicatrisation [107].

Le praticien doit pouvoir évaluer s'il existe une relation entre l'importance de l'inflammation et la quantité de plaque et les facteurs de rétention de plaque (tartre, restaurations débordantes, lésions carieuses non traitées). Ce rapport peut aider le praticien à déterminer la " susceptibilité » du patient vis-à-vis de l'agression bactérienne.

L'examen du parodonte doit objectiver :

- L'inflammation, qui se traduit par une modification de couleur (érythème), de volume (œdème ou hyperplasie) et une augmentation de la tendance au saignement (au brossage, à la mastication ou spontané). L'inflammation traduit l'importance de la réaction du tissu gingival à la plaque bactérienne supragingivale.

- L'œdème, qui est le résultat d'une extravasation de liquide intravasculaire dans le compartiment extracellulaire du tissu conjonctif gingival. Les tissus prennent alors un aspect lisse et luisant, en commençant le plus souvent aux papilles qui émergent partiellement des espaces interdentaires.

- $\quad$ L'indice de plaque de Silness et Loë (index validé)

- 0 : pas de plaque

- 1 : mince film de plaque au contact de la gencive marginale visible seulement après exploration à la sonde

- 2 : accumulation modérée de plaque au contact de la gencive marginale ; pas de plaque dans les espaces interdentaires; dépôts visibles à l'œil nu

- 3 : grande accumulation de plaque au contact de la gencive marginale ; présence de plaque dans les espaces interdentaires

- Le sondage permet de mettre en évidence deux paramètres importants : la profondeur de poche et la perte d'attache. II renseigne sur la gravité des lésions provoquées par la maladie parodontale mais sert également de guide et de repère thérapeutique lors des phases de 
réévaluation et de maintenance. On considère qu'un site est sain lorsque la profondeur de poche n'excède pas 2,5 à $3 \mathrm{~mm}$.

- Le saignement au sondage : le sondage d'un sulcus sain avec une sonde mousse n'entraîne pas de saignement. Le saignement au sondage fournit le meilleur critère diagnostique de I'inflammation gingivale; s'il ne prouve pas l'activité d'une lésion, son absence semble en revanche être significative de la stabilité dans le temps de la lésion observée [108].

- $\quad$ L'indice gingival de Loë et Silness (index validé)

- 0 : aucun signe d'inflammation

- 1 : modification de couleur

- 2 : inflammation visible à l'œil nu et tendance au saignement au passage de la sonde

- 3 : inflammation importante et tendance au saignement spontané

- Une suppuration : suintement ou écoulement de pus qui peut apparaître soit au sondage, soit lorsque le praticien exerce une pression digitale sur la gencive libre. C'est le signe tardif d'une infection parodontale. II faut nécessairement établir ici un diagnostic différentiel avec une lésion d'origine endodontique (vitalité pulpaire, signes cliniques d'inflammation gingivale, signes radiologiques).

- La présence de lésions inter-radiculaires. Cet examen se fait à l'aide d'une sonde de Nabers pour mettre en évidence les zones de séparation radiculaire : la perte osseuse horizontale est mesurée, ce qui permet de répartir les lésions selon la classification de Hamp et al. [109].

- Classe I : lyse osseuse horizontale inférieure à $3 \mathrm{~mm}$

- Classe II : lyse osseuse horizontale supérieure à $3 \mathrm{~mm}$ non transfixante

- Classe III : lyse osseuse transfixante

Recommandations proposées: Un bilan à la recherche de FIBD doit impérativement comprendre un examen clinique (interrogatoire, sondage parodontal, tests de vitalité, percussion, palpation des chaînes ganglionnaires...) (APF). Lors de l'examen clinique chez les patients à haut risque d'endocardite infectieuse, le sondage parodontal doit être réalisé sous antibioprophylaxie (APF).

\subsection{Examen radiologique}

Il a pour but de rechercher ou de vérifier l'existence d'une pathologie évidente ou supposée lors de l'examen clinique. II précise l'étendue et le type de lésion (caries dentaires, lésions apicales, apex ou racines résiduels, dents incluses, corps étrangers).

L'orthopantomogramme (OTP ou radiographie panoramique) constitue souvent un examen de première intention. Il permet en effet d'obtenir, de façon simple et rapide, une image globale de toutes les structures dento-alvéolaires. La radiographie panoramique dentaire complète l'examen clinique. Elle parait indispensable au bilan initial et renseigne notamment sur la présence de : dents incluses, dystopies, dysplasies, racines ou kystes résiduels, caries, granulomes, parodontopathies et traitements endodontiques. 
Les clichés endobuccaux (rétroalvéolaires ou rétrocoronaires) complètent l'orthopantomogramme, avec une supériorité pour objectiver l'intégrité du parodonte (lamina dura, épaisseur ligamentaire, alvéolyse), de la racine, la qualité de l'obturation canalaire et la présence de lésions apicales.

Dans une étude prospective, Bishay et al. [110] en 1999 ont comparé chez 65 patients devant bénéficier d'une greffe de cellules souches hématopoïétiques, l'apport de la radiographie panoramique dentaire avec celui du bilan rétroalvéolaire long cône. Ce dernier a permis la détection de façon significative des caries, des lésions parodontales et des défauts dans les restaurations coronaires. Ils n'ont pas trouvé de différence pour la détection de foyers péri-apicaux. En revanche, la radiographie panoramique s'est révélée significativement meilleure pour détecter les dents incluses. Ces auteurs concluent que les deux examens sont complémentaires et qu'ils doivent être employés conjointement dans le bilan radiologique des patients à risque.

Selon les recommandations de la HAS pour la prescription d'examens radiologiques [111]:

- Avant radiothérapie ou chimiothérapie, la mise en état bucco-dentaire nécessite une radiographie panoramique qui complète l'examen clinique et renseigne sur la présence de dents incluses, de racines ou de kystes résiduels et la valeur des traitements endodontiques. Des radiographies rétro-alvéolaires peuvent permettre d'affiner l'examen panoramique (NP 3).

- En règle générale, pour toute recherche de foyer infectieux, dans le cadre d'une pathologie générale ou avant un acte chirurgical, un bilan d'imagerie dentaire complet sera réalisé (NP 2).

En cas de doute sur l'existence d'un foyer péri-apical, un examen tomodensitométrique pourra être prescrit. Dans une étude expérimentale chez l'animal, Jorge et al. [112] ont en effet montré que la tomodensitométrie était capable de mettre en évidence des lésions péri-apicales avant qu'elles ne soient visibles à l'examen radiologique conventionnel.

Recommandations proposées : Un bilan initial à la recherche de FIBD doit impérativement comprendre un examen radiographique panoramique (APF). En cas de doute à la lecture de l'examen panoramique, l'examen radiographique doit être complété par d'autres examens : clichés rétro-alvéolaires, tomographie volumique à faisceau conique (cone beam), tomodensitométrie (scanner) (APF).

\subsection{Bilan biologique}

La numération formule sanguine fait partie du bilan général infectieux, ainsi que le dosage de certaines protéines plasmatiques (CRP, procalcitonine). Mais s'il permet d'attirer l'attention sur un syndrome inflammatoire sans spécificité, le bilan biologique ne peut en affirmer l'origine dentaire ou parodontale.

\section{Quelles stratégies thérapeutiques adopter?}

L'ensemble des études retrouvées par la recherche documentaire montre une grande disparité quant aux conduites à tenir proposées vis-à-vis des FIBD. Devant cette hétérogénéité des données de la 
littérature, il a été demandé au groupe de cotation de se prononcer sur la gestion des foyers infectieux chez les patients à risque.

Recommandations proposées :

\section{Population générale}

Pour l'ensemble de la population, indépendamment de toute notion de pathologie:

- Il est recommandé d'éliminer les FIBD (APF).

- Il est recommandé d'éliminer les SRIP (CR).

\section{Information}

La découverte d'un FIBD doit faire l'objet d'une information délivrée au patient (APF).

Cette information doit préciser les conséquences éventuelles de cette infection (APF).

La découverte d'une SRIP doit faire l'objet d'une information délivrée au patient (APF).

Cette information doit :

- contenir l'évaluation du risque de développement d'une infection (CR)

- préciser les conséquences éventuelles de cette infection (APF).

\section{Élimination des foyers infectieux}

Par rapport au patient sain, chez qui il est recommandé d'éliminer les foyers infectieux, il est particulièrement recommandé d'éliminer les FIBD (APF) :

- Avant une chimiothérapie anticancéreuse non aplasiante, dans la mesure où l'urgence à instaurer le traitement l'y autorise

- Chez les sujets à risque modéré d’EI

- Avant un traitement par bisphosphonates (quelle qu'en soit l'indication)

- Chez les patients présentant des pathologies respiratoires chroniques (bronchopneumopathie obstructive chronique, asthme ...)

- Chez les patients diabétiques équilibrés

- Chez une femme ayant un projet de grossesse.

Il est impératif d'éliminer les FIBD :

- Avant une transplantation ou l'instauration d'un traitement immunosuppresseur, dans la mesure où l'urgence à instaurer le traitement l'y autorise (APF)

- Avant une chimiothérapie aplasiante, dans la mesure où l'urgence à instaurer le traitement l'y autorise (APF) 
- Avant une radiothérapie cervico-faciale (FIBD situés dans les faisceaux d'irradiation) (APF)

- Chez les sujets à haut risque d'El (APF)

- Avant la mise en place de prothèse articulaire (CR)

- Chez les patients diabétiques non équilibrés (hémoglobine glyquée > $7 \%$ ) (CR)

Attitude générale chez les patients présentant un risque infectieux particulier (autre que dentaire) :

$$
\text { Évaluation du risque }
$$

Un contact préalable avec le médecin responsable du suivi du patient est recommandé pour évaluer le risque médical (APF).

\section{Urgence infectieuse}

En cas de survenue d'une d'infection bucco-dentaire, le contexte médical général (radiothérapie, chimiothérapie, chirurgie cardiaque ...) ne doit pas retarder la prise en charge chirurgicale et/ou médicale de l'urgence infectieuse. En particulier, un abcès doit être drainé (APF).

\section{Décision thérapeutique}

Dans les cas de FIBD comme dans les SRIP, le choix de la thérapeutique bucco-dentaire doit intégrer des notions multiples, en particulier (APF) :

- le pronostic vital du patient lié à l'affection générale

- le risque lié à l'abstention thérapeutique, en fonction notamment de la virulence présumée du foyer infectieux

- la morbidité inhérente à chaque solution thérapeutique

- le bénéfice attendu de la proposition thérapeutique pour le confort de vie du patient

- l'observance prévisible du patient aux manœuvres d'hygiène et aux visites de contrôle

Dans tous les cas, les différents traitements doivent être expliqués au patient et son consentement éclairé doit être recueilli (APF).

\section{Cicatrisation}

La cicatrisation muqueuse après avulsion dentaire nécessite une durée minimale d'une semaine. Elle doit être évaluée par un contrôle clinique (APF).

\subsection{Immunodépression}

Globalement, la littérature scientifique n'est pas unanime sur ce sujet. Deux attitudes s'opposent: adopter chez les patients à haut risque infectieux du fait de l'immunodépression les mêmes pratiques que pour la prophylaxie de l'El, ou proposer des attitudes plus nuancées en fonction du terrain. Pour la plupart, ces études reposent uniquement sur des accords professionnels [6]. 


\subsubsection{Chimiothérapie}

Du fait des impératifs de la lutte contre la maladie, le temps disponible avant la première cure est souvent très réduit et ne permet pas de pratiquer l'ensemble des thérapeutiques bucco-dentaires nécessaires [113]. Ce manque de temps oblige à traiter avec priorité les lésions qui paraissent les plus virulentes.

Une enquête pour évaluer les pratiques professionnelles a été réalisée auprès de 132 membres de l'American Academy of Maxillofacial Prosthetics [114]. $68 \%$ ont répondu au questionnaire. L'ensemble des praticiens s'accordait sur la nécessité d'un bilan avant la chimiothérapie et sur la distinction entre affections aiguës et chroniques. Les lésions périapicales d'origine endodontique devaient être traitées le plus souvent par une reprise de traitement, alors que les dents présentant une parodontite sévère devaient quant à elles, être avulsées. Néanmoins la décision de conservation d'une dent était adaptée en fonction de sa valeur stratégique.

Toljanic et al. [115] ont évalué dans une étude prospective le bilan et le traitement dentaire avant une chimiothérapie aplasiante; 48 patients ont été inclus dans l'étude. Les infections bucco-dentaires chroniques étaient classées en affections faibles, modérées ou sévères en fonction de la probabilité de survenue d'un accident infectieux aigu pendant la chimiothérapie. $79 \%$ des patients présentaient au moins une lésion chronique et $44 \%$ une pathologie sévère susceptible de se compliquer pendant les cures. La durée moyenne d'hospitalisation était de 37 jours (entre 17 et 125 jours). Pendant cette période, deux patients ont eu un abcès qui a été traité par une antibiothérapie sans interrompre la chimiothérapie. Les auteurs concluent à la possibilité d'administrer une chimiothérapie chez des patients présentant une affection chronique sans risque supplémentaire. Cette attitude permettrait un traitement plus conservateur pour les patients devant bénéficier d'une chimiothérapie.

Les lésions endodontiques asymptomatiques s'aggravent rarement pendant la chimiothérapie [116], à l'inverse des lésions parodontales [117-120].

Dans le cadre d'une neutropénie sévère (polynucléaires neutrophiles $<1000 / \mathrm{mm}^{3}$ ) l'American Academy of Pediatric Dentistry recommande une antibioprophylaxie avant un geste invasif [121].

Enfin, plusieurs études s'accordent sur le fait que le traitement bucco-dentaire avant une chimiothérapie permettrait de réduire les épisodes fébriles et les infections pendant les cures [118, 122-124].

Recommandations proposées :

\section{Avant la chimiothérapie}

Dans la mesure où l'urgence à instaurer le traitement I'y autorise, un bilan bucco-dentaire doit être entrepris au plus tôt avant le début d'une chimiothérapie anticancéreuse (APF).

Les gestes chirurgicaux destinés à assainir la cavité buccale doivent être entrepris au plus tôt de façon à ce que la cicatrisation muqueuse soit acquise avant le début de la chimiothérapie (APF). 


\section{Pendant la chimiothérapie}

Les gestes thérapeutiques invasifs (avulsion dentaire ...) doivent être pratiqués :

- en connaissance du bilan biologique (hémogramme, hémostase) (APF)

- seulement s'ils sont urgents (CR)

- sous antibioprophylaxie poursuivie jusqu'à la cicatrisation muqueuse de la plaie si le taux de polynucléaires neutrophiles (PN) est inférieur à $500 / \mathrm{mm}^{3}$ de sang (APF) (pas de consensus pour une antibioprophylaxie pour un taux de PN supérieur à $500 / \mathrm{mm}^{3}$ ) (PC).

En dehors des cas d'urgence, les gestes chirurgicaux peuvent être pratiqués dans une phase de normalité des polynucléaires neutrophiles (APF).

\subsubsection{Thérapeutiques biologiques ciblées}

Des recommandations ${ }^{*}$ ont été publiées par le Club Rhumatisme et Inflammation (CRI : section spécialisée de la Société Française de Rhumatologie).

Recommandations du CRI concernant les soins bucco-dentaires :

\section{Anti-TNF alpha}

Il est recommandé une hygiène bucco-dentaire et des soins réguliers. En cas d'état buccodentaire défectueux, des soins appropriés devront être effectués avant de débuter un traitement par anti-TNF alpha.

Soins conservateurs

II n'y a pas d'éléments justifiant l'arrêt des anti-TNF alpha.

Soins à risque infectieux (avulsion, drainage d'abcès...)

Il est recommandé d'arrêter les anti-TNF alpha et de proposer une antibioprophylaxie.

Pose d'implants

II n'y a pas d'indication formelle à arrêter les anti-TNF alpha, tout en restant vigilant aux risques infectieux potentiels.

\section{Situations pratiques}

En cas d'intervention chirurgicale ou de soins bucco-dentaires programmés, des mesures prophylactiques sont justifiées, en particulier l'arrêt des anti-TNF alpha, dans des délais suffisants pour limiter les risques infectieux. Contacter le médecin prescripteur de l'anti-TNF alpha.

Durée d'arrêt des anti-TNF alpha avant chirurgie :

- etanercept : au moins 2 semaines

- infliximab : au moins 4 semaines

- adalimumab : au moins 4 semaines

\footnotetext{
* Club Rhumatismes et inflammations. Fiches pratiques. http://www.cri-net.com (consulté le 16/07/2011)
} 


\section{Rituximab}

Il est recommandé une hygiène bucco-dentaire et des soins réguliers. En cas d'état buccodentaire défectueux, des soins appropriés devront être effectués avant de débuter un traitement par rituximab.

Soins à risque infectieux (avulsion, drainage d'abcès...)

Non réalisation de la $2^{\text {ème }}$ perfusion de rituximab si le soin doit intervenir entre 2 perfusions. Mais le plus souvent, le médicament ne pourra pas être arrêté car le cycle de deux perfusions aura été réalisé avec des conséquences sur l'immunité pendant au moins 6 mois. II est recommandé de proposer une antibioprophylaxie.

Implants

II n'y a pas de précaution particulière à prendre tout en restant vigilant sur la survenue potentielle d'infections.

\section{Abatacept}

Compte tenu des données de tolérance qui rapportent une plus grande fréquence des événements indésirables graves de nature infectieuse dans le groupe abatacept que dans le groupe placébo ( $3 \%$ vs. 1,9\%), il est recommandé une hygiène bucco-dentaire et des soins réguliers. En cas d'état bucco-dentaire défectueux, des soins appropriés devront être effectués avant de débuter un traitement par abatacept. Pour les soins conservateurs, on peut proposer une antibioprophylaxie. Pour les soins à risque infectieux (avulsion, drainage d'abcès ...), il est recommandé de décaler la perfusion de l'abatacept et de proposer une antibioprophylaxie.

\section{Tocilizumab}

Il est recommandé une hygiène bucco-dentaire et des soins réguliers. En cas d'état buccodentaire défectueux, des soins appropriés devront être effectués avant de débuter un traitement par tocilizumab

Soins conservateurs

Il n'y a pas d'éléments justifiant l'arrêt du tocilizumab.

Soins à risque infectieux (avulsion, drainage d'abcès...)

Il est recommandé d'arrêter le tocilizumab avant les soins dentaires, au moins 4 semaines, et de proposer une antibioprophylaxie.

Implants

Il n'y a pas d'indication formelle à arrêter le tocilizumab tout en restant vigilant aux risques infectieux potentiels.

Situation pratique

En raison d'un possible retard de cicatrisation dû au tocilizumab et de sa capacité à masquer des signes infectieux post-opératoires (absence de fièvre et CRP normale), il est recommandé de retarder la chirurgie programmée en respectant un délai d'au moins 4 semaines après la dernière perfusion de tocilizumab. 
Recommandations proposées : Il est impératif d'éliminer les FIBD avant instauration d'une thérapie biologique ciblée à visée immunosuppressive (APF). Si des soins invasifs (avulsions dentaires, pose d'implants...) sont envisagés pendant le traitement, une attention particulière doit être portée à la survenue potentielle d'infections post-opératoires : vigilance, information du patient. La décision thérapeutique est prise au cas par cas en pesant le rapport bénéfice/risque avec le médecin prescripteur qui propose éventuellement une mise en condition du patient (pouvant comprendre la suspension du traitement) (APF).

Pour davantage de précision se reporter aux recommandations du CRI.

\subsubsection{Transplantations}

Meyer et al. [125] ont étudié les infections dentaires et les rejets de transplantation cardiaque chez 74 patients. Ils ont comparé deux groupes de patients avant la transplantation cardiaque, l'un avec un traitement radical (extractions de toutes les dents non vitales), l'autre sans traitement. Aucune différence significative n'a été observée entre les deux groupes en termes de mortalité, de rejet de greffe ou d'infection. Ils ont conclu qu'en cas de défaillance cardiaque sévère, les patients ne nécessitaient pas un traitement bucco-dentaire pré-opératoire strict.

Recommandation proposée : Les gestes chirurgicaux destinés à assainir la cavité buccale doivent être entrepris au plus tôt, de façon à ce que la cicatrisation muqueuse soit acquise avant la transplantation ou l'instauration d'un traitement immunosuppresseur (APF).

\subsection{Affections cardio-vasculaires}

Dans une étude cas témoin chez 21 patients, Lockhart et al. [126] ont pratiqué des extractions dentaires en même temps qu'une chirurgie valvulaire, sous couvert d'une antibioprophylaxie. Aucune complication opératoire n'a été rapportée. Cette étude relativise le danger lié à la bactériémie iatrogène d'origine bucco-dentaire.

Recommandations proposées : Un bilan bucco-dentaire doit être entrepris au plus tôt avant chirurgie valvulaire (APF). Les gestes chirurgicaux destinés à assainir la cavité buccale doivent être entrepris au plus tôt de façon à ce que la cicatrisation muqueuse soit acquise avant la chirurgie valvulaire (APF)

\subsection{Radiothérapie cervico-faciale}

Il est unanimement admis qu'une irradiation cervico-faciale thérapeutique doit toujours être précédée d'un examen et d'une mise en état préalable de la denture. Ce bilan doit être réalisé dès la programmation de la radiothérapie pour la prévention " primaire " et "secondaire " des infections postradiques [127]. En ce qui concerne l'attitude à tenir, "l'édentation systématique et définitive ", qui a été la règle jusqu'aux années 1970 [128] doit maintenant être proscrite.

Indications de la conservation des dents situées dans les faisceaux d'irradiation [129]

Les bonnes pratiques avant radiothérapie relèvent toujours d'un compromis qui tient compte de paramètres connus, tels la dose d'irradiation et l'état bucco-dentaire, et de paramètres plus difficilement contrôlables, comme l'observance du patient. Les buts poursuivis sont la prévention des complications et la préservation de la qualité de vie du patient (sa réhabilitation masticatoire, phonétique et esthétique). Seules des dents jugées saines ou correctement traitées au préalable peuvent être conservées. Doivent être extraites avant de commencer l'irradiation : les dents délabrées, les dents présentant des foyers infectieux apicaux ou parodontaux de mauvais pronostic ; les dents qui ultérieurement vont s'avérer inutilisables d'un point de vue prothétique (dents en 
malposition, versées ou égressées, etc.) ; les dents qui constituent une source de traumatisme pour les muqueuses linguales ou labio-jugales en regard ; les dents en désinclusion avec antécédents de péricoronarite. L'avulsion des dents incluses doit être discutée en tenant compte du délai de cicatrisation qui est nécessaire (au moins trois à quatre semaines) et de l'« urgence » qu'il y a à débuter l'irradiation. Pour toutes ces raisons, les dents asymptomatiques sont généralement laissées en place.

La conservation de dents dans le secteur irradié nécessite une excellente motivation du patient (hygiène, fluoroprophylaxie, surveillance).

Une étude de Epstein et al. [130] en 1998, a montré que la perte d'attache parodontale était plus importante après une radiothérapie cervico-faciale, même avec de faibles doses. Les auteurs ont conclu que le bilan pré-radiothérapie devait tenir compte de cette considération susceptible de modifier l'attitude thérapeutique.

Pour information, on présente ci-dessous le schéma des préconisations sur la conduite à tenir en cas de soins bucco-dentaires après radiothérapie, selon les Référentiels inter-régionaux en Soins Oncologiques de Support 2011 [131], insistant sur la nécessité d'une poursuite de l'antibioprophylaxie jusqu'à cicatrisation muqueuse.

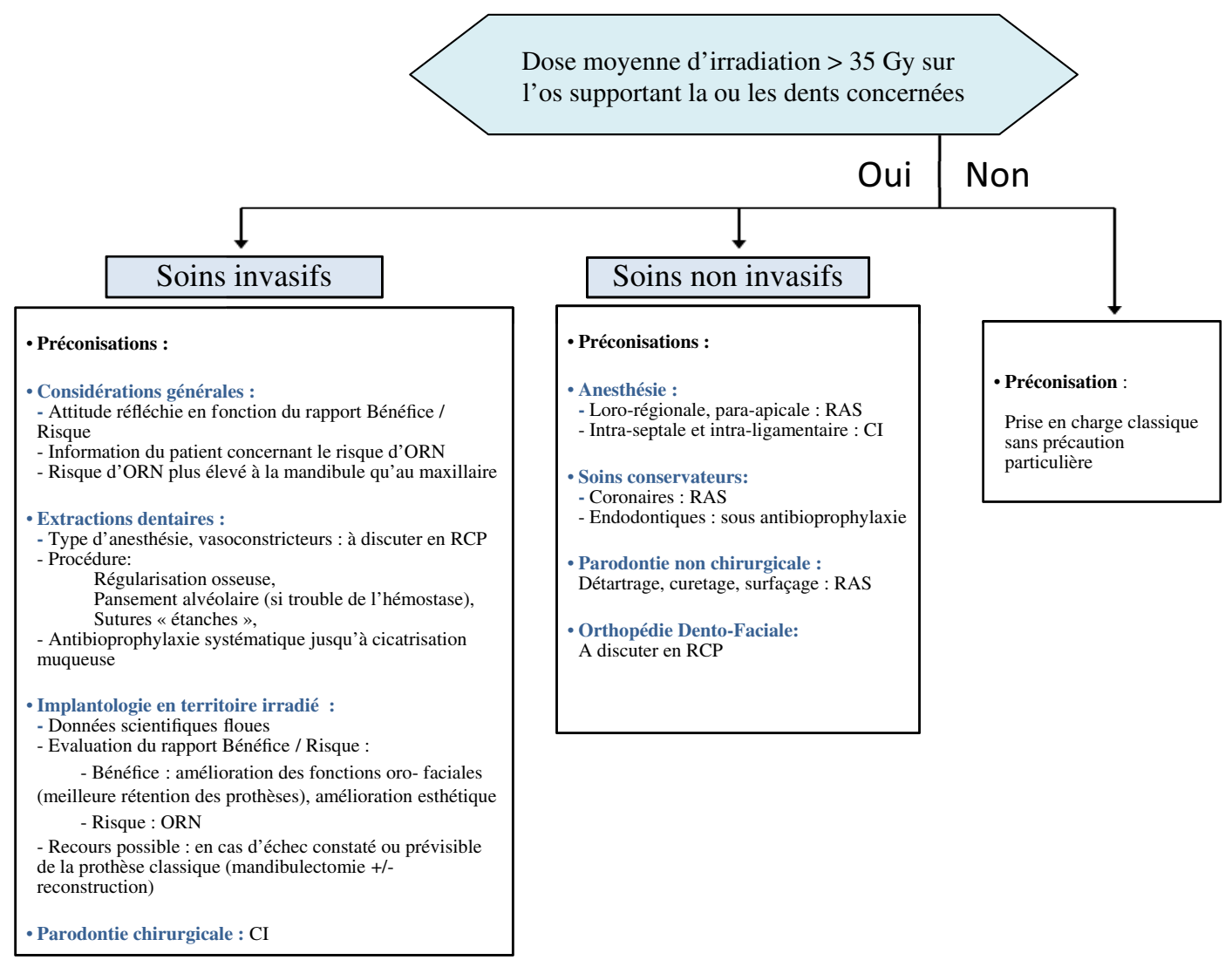


Recommandations proposées :

\section{Avant la radiothérapie}

Les gestes chirurgicaux destinés à assainir la cavité buccale doivent être entrepris au plus tôt de façon à ce que la cicatrisation muqueuse soit acquise avant le début de la radiothérapie (APF).

Après la radiothérapie (et quel que soit le délai)

Il n'y a pas lieu de prendre des mesures particulières si la dose d'irradiation reçue par le maxillaire et/ou la mandibule est inférieure à $30 \mathrm{~Gy}(\mathrm{CR})$.

Afin de diminuer le risque d'ostéoradionécrose, les avulsions dentaires éventuellement nécessaires doivent être effectuées :

- $\quad$ après information sur la dose reçue et les faisceaux (champs) d'irradiation (APF)

- dans un plateau technique chirurgical adapté à la situation et apportant les garanties de qualité et de sécurité (APF).

Devant un risque d'ostéoradionécrose, un traitement antibiotique doit être institué en cas de geste invasif (avulsion dentaire, curetage...) (APF). Il convient de débuter cette prescription au moins $1 \mathrm{~h}$ avant le geste (APF) (pas de consensus pour un délai plus long) et de la poursuivre jusqu'à la cicatrisation de la muqueuse (APF).

\subsection{Traitements par bisphosphonates}

Selon les recommandations AFSSAPS $2007^{*}$ :

Dans le cadre de pathologies malignes, il est préférable de ne débuter le traitement par bisphosphonates, si l'état clinique du patient le permet, qu'une fois la situation dentaire assainie et dans la mesure du possible, après cicatrisation osseuse complète (120 jours).

Avant la mise en place du traitement, les racines résiduelles, les dents à mauvais pronostic et les dents en désinclusion doivent être extraites. De même, les foyers de parodontite ou de péri-implantite devront être assainis.

* Recommandations sur la prise en charge bucco-dentaire des patients traités par bisphosphonates (19/12/2007), http://www.afssaps.fr/Infos-de-securite/Lettres-aux-professionnels-de-sante/Recommandations-sur-la-prise-en-chargebucco-dentaire-des-patients-traites-par-bisphosphonates (consulté le 12/09/2009) 
En cours de traitement, seules les dents ayant une mobilité de stade 3 ou présentant un foyer infectieux actif doivent être extraites. Ces interventions se font sous traitement antibiotique d'au moins 10 jours.

Recommandations proposées : Chez les patients traités ou ayant été traités par bisphosphonates dans le cadre d'une pathologie maligne, il est recommandé de pratiquer les avulsions dans un plateau technique chirurgical adapté à la situation et apportant les garanties de qualité et de sécurité (APF). Chez les patients traités ou ayant été traités par des bisphosphonates en dehors du cadre d'une pathologie maligne, les avulsions peuvent faire l'objet d'une prise en charge en cabinet d'omnipratique dentaire, dans le respect des règles de prise en charge $(C R)$.

\subsection{Diabète sucré}

Il n'a pas été trouvé de consensus sur le fait de savoir si les patients diabétiques équilibrés (hémoglobine glyquée $<7 \%$ ) nécessitent des précautions particulières quant à la gestion des FIBD du seul fait de leur diabète (PC).

Recommandations proposées : Les patients diabétiques peuvent faire l'objet d'une prise en charge en cabinet d'omnipratique dentaire, après vérification de l'équilibre du diabète (APF).Chez les patients diabétiques non équilibrés (hémoglobine glyquée $>7 \%$ ), en cas de geste invasif, il faut commencer le traitement antibiotique prophylactique dans l'heure qui précède le geste et le poursuivre jusqu'à cicatrisation muqueuse de la plaie (CR).

\subsection{Prothèses articulaires}

Dans une étude prospective cas-contrôle réalisée chez 339 patients, Berbari et al. [132] ont montré que les soins dentaires, quelle que soit la procédure de soin, ne représentent pas un risque particulier pour les patients porteurs de prothèse de hanche ou de genou. Dans cette étude, 339 patients ont été hospitalisés entre décembre 2001 et avril 2006 pour une infection de prothèse totale de genou ou de hanche. Les 339 patients contrôle avaient également une prothèse de genou ou de hanche, mais sans infection connue. Les facteurs de risque vis-à-vis de l'infection de prothèse, y compris les actes buccodentaires, dans les 2 ans avant le diagnostic d'infection ont été colligés. Les actes bucco-dentaires étaient classés en risque faible (orthodontie, traitement fluoré) ou élevé (traitement parodontal, avulsion). L'âge moyen des patients était de 69,5 ans et l'ancienneté de la prothèse de 15,5 mois. L'âge moyen des patients contrôle était de 71,4 ans et l'ancienneté de la prothèse de 49,9 mois. Les paramètres significativement associés à l'infection prothétique étaient : le diabète, les antécédents d'arthroplastie et l'immunodépression. L'antibiothérapie prophylactique avant un acte bucco-dentaire n'était pas différente entre les deux groupes de patients. II en résultait que, pour les gestes à faible risque, 41 patients infectés et 65 patients contrôle n'avaient pas eu d'antibiotiques (OR 0,6 ; IC 95 \% 0,4-1,1) et 59 patients infectés et 87 patients contrôle avaient eu des antibiotiques (OR 0,8 ; IC 95 \% 0,5-1,2) tandis que, pour les gestes à risque élevé, respectivement 33 et 49 n'avaient pas eu d'antibiotiques, et 95 et 148 en avaient eu (OR 0,8 et 0,7, respectivement) [6]. Les auteurs ont conclu 
qu'une antibioprophylaxie ne permet pas de diminuer le risque d'infection sur prothèse articulaire et que de ce fait celle-ci doit être abandonnée.

Les données actuelles de la littérature montrent donc qu'il n'existe que très peu de lien entre les infections articulaires et les procédures dentaires; de plus, une antibioprophylaxie n'est pas susceptible de réduire le risque d'infection sur prothèse articulaire. De ce fait il n'y a pas lieu de proposer une antibioprophylaxie du simple fait de la présence d'une prothèse articulaire.

Des précautions peuvent toutefois être prises avant la mise en place de la prothèse articulaire.

Recommandation proposée : Les gestes chirurgicaux destinés à assainir la cavité buccale doivent être entrepris au plus tôt de façon à ce que la cicatrisation muqueuse soit acquise avant la pose de la prothèse articulaire (APF).

\section{Quelles sont les mesures de suivi à envisager ?}

Chez les patients présentant un risque majoré d'infection à point de départ dentaire ou parodontal, une surveillance au long cours parait indispensable. Les données de la littérature sur les modalités de ce suivi varient, y compris à l'intérieur des différentes catégories de patients. A titre indicatif, pour les patients indemnes de toute pathologie connue, il est habituellement recommandé un contrôle annuel chez le chirurgien-dentiste associé à des mesures d'hygiène bucco-dentaire quotidienne.

La conférence de consensus de 2002 sur la prévention de l'EI, insiste dans sa recommandation sur la nécessité d'une surveillance bisannuelle : « une surveillance systématique de l'état bucco-dentaire doit être au minimum réalisée deux fois par an chez les patients porteurs de cardiopathie » [133].

Guillaud et al. [134] ont montré, sur deux années consécutives et pour la population à risque d’EI, que le pourcentage de patients ayant un suivi bucco-dentaire est faible $(48,1 \%)$, et que ce pourcentage apparaît encore moindre dans le sous-groupe des patients à haut risque d’EI (43,2 \%). Le suivi buccodentaire semble diminuer avec l'âge du patient. Un faible niveau de suivi bucco-dentaire chez des patients à risque d'El est également relevé, dans l'étude de De Geest et al. [135], en Belgique où seulement un tiers des patients dentés avait un suivi annuel associé à un brossage quotidien et où pratiquement aucun suivi n'était noté chez les patients édentés. Pour Gutschik et Lippert [136], au Danemark, seulement $50 \%$ des patients avec prothèse valvulaire avaient des visites bucco-dentaires régulières. Barreira et al. [137] insistent, quant à eux, sur la prévention avec la nécessité de prendre en charge les enfants à risque d'El. Le suivi bi-annuel recommandé pour les patients à risque d'El ne semble donc malheureusement pas appliqué.

L'amélioration de l'hygiène bucco-dentaire est capitale afin de limiter les bactériémies spontanées survenant à la suite de manœuvres de mastication et de brossage de dents. Cela implique une vérification régulière de l'état bucco-dentaire par un spécialiste de la cavité buccale, au minimum deux fois par an, chez les patients présentant une cardiopathie à haut risque d’EI [138].

Après radiothérapie, la consultation bucco-dentaire s'effectue généralement au même rythme que la surveillance médicale, en général tous les 3 mois pendant 1 an, puis tous les 6 mois pendant 2 à 3 ans, puis tous les ans. En pratique, un examen panoramique peut être réalisé tous les 6 mois la 1ère année puis tous les ans par la suite, afin de rechercher des signes de lésions infectieuses et de dystrophie osseuse (premiers signes de l'ostéoradionécrose). 
Dans une étude rétrospective comparant le protocole de prise en charge bucco-dentaire avant et après radiothérapie de deux groupes suivis à 12 ans d'intervalle (1993 et 2005), Sernnhenn-Kirchner et al. [139] montrent une amélioration significative du suivi de ces patients. Le groupe suivi en 2005 a bénéficié d'une consultation pour un bilan bucco-dentaire systématique avant l'irradiation ainsi que la mise en place de gouttières de fluoration. Les patients présentaient moins de caries après la radiothérapie, moins de candidoses et un édentement moins important qu'en 1993. Le traitement avant radiothérapie était également plus conservateur en 2005 qu'en 1993.

Une étude de Katsura et al. [140] en 2008 a montré que le risque d'ostéoradionécrose augmentait lorsque le patient présentait des poches parodontales supérieures à $5 \mathrm{~mm}$, un score de plaque > à $40 \%$ et une alvéolyse supérieure à $60 \%$. Les auteurs recommandent donc une surveillance régulière avec une thérapeutique parodontale de soutien.

Le groupe de travail de la Société Française d'ORL (2005) n'a pas trouvé de consensus sur le rythme des contrôles dentaires, mais a proposé un examen dentaire biannuel, en se basant sur un accord professionnel [141].

Après chimiothérapie, il n'y a pas lieu d'adopter, vis-à-vis de la recherche de FIBD, une attitude différente de celle proposée à un patient sain.

Dans le cadre des recommandations de l'AFSSAPS ${ }^{*}$, un contrôle bucco-dentaire doit être effectué tous les 4 mois pour les patients recevant des bisphosphonates dans le cadre de pathologie maligne, et au moins une fois par an chez ceux qui prennent ces médicaments dans le cadre de l'ostéoporose ou la maladie de Paget. Marx et al. [142] recommandent le même suivi.

Dans le cas des transplantations d'organe, le risque d'infection est majeur dans les 3 à 6 mois qui suivent la transplantation. Durant cette période, seuls les soins d'urgence peuvent être entrepris. Au delà de 6 mois, les soins pourront se faire chez le praticien habituel. Une antibioprophylaxie sera discutée avec le spécialiste en charge du patient, en fonction de l'immunodépression [143].

Recommandations proposées :

Fréquence de suivi

Après la mise en état de la cavité buccale, à distance de la cicatrisation, une fois obtenue l'adhésion du patient aux méthodes d'hygiène, il doit être institué un suivi bucco-dentaire avec une fréquence supérieure à celle recommandée pour la population générale (annuelle) dans les situations suivantes (APF) :

- chez les sujets transplantés

\footnotetext{
* Recommandations sur la prise en charge bucco-dentaire des patients traités par bisphosphonates (19/12/2007), http://www.afssaps.fr/Infos-de-securite/Lettres-aux-professionnels-de-sante/Recommandations-sur-la-prise-en-chargebucco-dentaire-des-patients-traites-par-bisphosphonates (consulté le 12/09/2009)
} 
- chez les patients à haut risque d'El

- après irradiation thérapeutique supérieure à 30 Gy

- chez les patients traités ou ayant été traités par bisphosphonates dans le cadre d'une pathologie maligne

- chez les patients diabétiques non équilibrés (hémoglobine glyquée > $7 \%$ )

- chez les patients atteints d'un SIDA.

Dans ces cas, il convient d'instituer une fréquence de suivi bucco-dentaire de 4 à 6 mois (CR).

Nota : il a été acté le fait de suggérer une fréquence plus importante que la nécessité clinique, compte tenu d'une faible observance prévisible.

En revanche :

- chez le sujet porteur de prothèse articulaire

- chez les sujets à risque modéré d'El

- chez les patients traités ou ayant été traités par bisphosphonates en dehors du cadre d'une pathologie maligne

la fréquence de suivi peut être identique à celle recommandée pour la population générale (annuelle) (CR).

Aucun consensus n'a été dégagé pour la fréquence de suivi dans affections respiratoires chroniques (bronchopneumopathie obstructive chronique, asthme...) (PC).

\section{Références}

1. Mascres C. L'infection focale d'origine dentaire. Encycl Med Chir Stomatologie 22043 C10. Elsevier Masson, Paris, 1988.

2. Roth D. Parodonte et infection focale. Rev Odontostomatol 1994;23:225-8.

3. Mouton C, Robert JC. Bactériologie bucco-dentaire. Masson, Paris, 1994.

4. Pontoriero R, Tonelli MP, Carnevale G, Mombelli A, Nyman SR, Lang NP. Experimentally induced periimplant mucositis. A clinical study in humans. Clin Oral Implants Res 1994;5:254-59.

5. Mombelli A, Lang NP. The diagnosis and treatment of peri-implantitis. Periodontol 2000 1998;17:63-76.

6. AFSSAPS. Prescription des antibiotiques en pratique bucco-dentaire. Paris, 2011.

7. Costello EK, Lauber CL, Hamady M, Fierer N, Gordon JI, Knight R. Bacterial community variation in human body habitats across space and time. Science 2009;326:1694-7.

8. Elder MJ, Stapleton F, Evans E, Dart JK. Biofilm-related infections in ophthalmology. Eye (Lond) 1995;9: 102-9.

9. Haffajee $A D$, Socransky SS. Introduction to microbial aspects of periodontal biofilm communities, development and treatment. Periodontol 2000 2006;42:7-12.

10. Debelian GJ, Olsen I, Tronstad L. Bacteremia in conjunction with endodontic therapy. Endod Dent Traumatol 1995;11:142-9.

11. Leonardo MR, Rossi MA, Silva LA, Ito IY, Bonifácio KC. EM evaluation of bacterial biofilm and microorganisms on the apical external root surface of human teeth. J Endod 2002;28:815-8.

12. Socransky SS, Haffajee AD, Cugini MA, Smith C, Kent RL. Microbial complexes in subgingival plaque. J Clin Periodontol 1998;25:134-44.

13. Thoden van Velzen SK, Abraham-Inpijn L, Moorer WR. Plaque and systemic disease: a reappraisal of the focal infection concept. J Clin Periodontol 1984;11:209-20. 
14. Okabe K, Nakagawa K, Yamamoto E. Factors affecting the occurrence of bacteremia associated with tooth extraction. Int J Oral Maxillofac Surg 1995;24:239-42.

15. Seymour RA, Lowry R, Whitworth JM, Martin MV. Infective endocarditis, dentistry and antibiotic prophylaxis; time for a rething ? Br Dent J 2000;189:610-6.

16. Kim B, Weiss LP. Dentally induced bacteremia and infection of total joint replacement arthroplasty. Focus Ohio Dent 1993;67:10-1.

17. Maestre JR, Mateo M, Sánchez P. Bacteremia after periodontal procedures. Rev Esp Quimioter 2008;21:153-6.

18. Bender IB, Montgomery S. Nonsurgical endodontic procedures for the patient at risk for infective endocarditis and other systemic disorders. J Endod 1986;12:400-7.

19. Guntheroth WG. How important are dental procedures as a cause of infective endocarditis? Am J Cardiol 1984;54:797-801.

20. American Dental Association,American Academy of Orthopaedics Surgeons. Antibiotic prophylaxis for dental patients with total joint replacements. J Am Dent Assoc 2003;134:895-9.

21. Forner $L$, Larsen $T$, Kilian $M$, Holmstrup P. Incidence of bacteremia after chewing, tooth brushing and scaling in individuals with periodontal inflammation. J Clin Periodontol 2006;33:401-7.

22. Bhanji S, Williams B, Sheller B, Elwood T, Mancl L. Transient bacteremia induced by toothbrushing a comparison of the Sonicare toothbrush with a conventional toothbrush. Pediatr Dent 2002;24:295-9.

23. Roberts GJ, Holzel HS, Sury MR, Simmons NA, Gardner P, Longhurst P. Dental bacteremia in children. Pediatr Cardiol 1997;18:24-7.

24. Roberts GJ, Jaffray EC, Spratt DA, Petire A, Greville C, Wilson M, Lucas VS. Duration, prevalence and intensity of bacteraemia after dental extractions in children. Heart 2006;92:1274-7.

25. Lockhart PB, Brennan MT, Sasser HC, Fox PC, Paster BJ, Bahrani-Mougeot FK. Bacteremia associated with toothbrushing and dental extraction. Circulation 2008;117:3118-25.

26. Shariff G, Brennan MT, Louise Kent M, Fox PC, Weinrib D, Burgess P, Lockhart PB. Relationship between oral bacteria and hemodialysis access infection. Oral Surg Oral Med Oral Pathol Oral Radiol Endod 2004;98:418-22.

27. American College of Chest Physicians/Society of Critical Care Medicine Consensus Conference: definitions for sepsis and organ failure and guidelines for the use of innovative therapies in sepsis. Crit Care Med 1992;20:864-74.

28. Seppanen L, Lauhio A, Lindqvist C, Suuronen R, Rautemaa R. Analysis of systemic and local odontogenic infection complications requiring hospital care. J Infect 2008;57:116-22.

29. Larkin EB, Scott SD. Metastatic paraspinal abscess and paraplegia secondary to dental extraction. $\mathrm{Br}$ Dent J 1994;177:340-2.

30. Brewer NS, MacCarty CS, Wellman WE. Brain abscess: a review of recent experience. Ann Intern Med 1975;82:571-6.

31. Roche M, Humphreys H, Smyth E, Phillips J, Cunney R, McNamara E, O'Brien D, McArdle O. A twelveyear review of central nervous system bacterial abscesses; presentation and aetiology. Clin Microbiol Infect 2003;9:803-9.

32. Colville A, Davies W, Heneghan M, Goodwin A, Griffiths T. A rare complication of dental treatment: Streptococcus oralis meningitis. Br Dent J 1993;175:133-4.

33. Crippin JS, Wang KK. An unrecognized etiology for pyogenic hepatic abscesses in normal hosts: dental disease. Am J Gastroenterol 1992;87:1740-3.

34. Tweedy CR, White WB. Multiple Fusobacterium nucleatum liver abscesses. Association with a persistent abnormality in humoral immune function. J Clin Gastroenterol 1987;9:194-7.

35. Scannapieco FA, Panesar M. Periodontitis and chronic kidney disease. J Periodontol 2008;79:1617-9.

36. Scannapieco FA, Bush RB, Paju S. Associations between periodontal disease and risk for nosocomial bacterial pneumonia and chronic obstructive pulmonary disease. A systematic review. Ann Periodontol 2003;8:54-69.

37. Lévêque L, Turcu A, Bielefeld $P$, Gresset $A C$, Duong $M$, Friedel J, Besancenot JF. Périartérite noueuse cutanée et infections dentaires. Rôle des streptocoques. Rev Med Interne 2001;22:992-6. 
38. Pischon N, Pischon T, Kröger J, Gulmez E, Kleber BM, Bernimoulin JP, Landau H, BrinkmannPG, Schlattmann P, Zernicke J, Buttgereit F, Detert J. Association among rheumatoid arthritis, oral hygiene, and periodontitis. J Periodontol 2008;79:979-86.

39. Ortiz P, Bissada NF, Palomo L, Han YW, Al-Zahrani MS, Panneerselvam A, Askari A. Periodontal therapy reduces the severity of active rheumatoid arthritis in patients treated with or without tumor necrosis factor inhibitors. J Periodontol 2009;80:535-40.

40. Al-Katma MK, Bissada NF, Bordeaux JM, Sue J, Askari AD. Control of periodontal infection reduces the severity of active rheumatoid arthritis. J Clin Rheumatol 2007;13:134-7.

41. Kielbassa AM, Hinkelbein W, Hellwig E, Meyer-Lückel H. Radiation-related damage to dentition. Lancet Oncol 2006;7:326-35.

42. Li Y, Taylor JM, Ten Haken RK, Eisbruch A. The impact of dose on parotid salivary recovery in head and neck cancer patients treated with radiation therapy. Int J Radiat Oncol Biol Phys 2007;67:660-9.

43. Nahmias C, Lemmens C, Faul D, Carlson E, Long M, Blodgett T, Nuyts J, Townsend D. Does reducing CT artifacts from dental implants influence the PET interpretation in PET/CT studies of oral cancer and head and neck cancer? J Nucl Med 2008;49:1047-52.

44. Chang DT, Sandow PR, Morris CG, Hollender R, Scarborough L, Amdur RJ, Mendenhall WM. Do preirradiation dental extractions reduce the risk of osteoradionecrosis of the mandible? Head Neck 2007;29:528-36.

45. Al-Nawas B, Grötz KA. Prospective study of the long term change of the oral flora after radiation therapy. Support Care Cancer 2006;14:291-6.

46. Münter MW, Karger CP, Hoffner SG, Hof H, Thilmann C, Rudat V, Nill S, Vannenmacher M, Debus J. Evaluation of salivary gland function after treatment of head-and-neck tumors with intensity-modulated radiotherapy by quantitative pertechnetate scintigraphy. Int J Radiat Oncol Biol Phys 2004;58:175-84.

47. Ortholan C, Chamorey E, Benezery K, Thariat J, Dassonville O, Poissonnet G, Bozec A, Follana P, Peyrade F, Sudaka A, Gerard JP, Bensadoun RJ. Modeling of salivary production recovery after radiotherapy using mixed models: determination of optimal dose constraint for IMRT planning and construction of convenient tools to predict salivary function. Int J Radiat Oncol Biol Phys 2009;73:178-86.

48. Ortholan C, Benezery K, Bensadoun RJ. Normal tissue tolerance to external beam radiation therapy: salivary glands. Cancer Radiother 2010;14:290-4.

49. Colella G, Cannavale R, Pentenero M, Gandolfo S. Oral implants in radiated patients: a systematic review. Int J Oral Maxillofac Implants 2007;22:616-22.

50. Ben Slama L, Hasni W, De Labrouhe C, Bado F, Bertrand JC. Osteoradionecrosis and dental implants. Rev Stomatol Chir Maxillofac 2008;109:387-91; discussion 391-2.

51. Ozen J, Dirican B, Oysul K, Beyzadeoglu M, Ucok O, Beydemir B. Dosimetric evaluation of the effect of dental implants in head and neck radiotherapy. Oral Surg Oral Med Oral Pathol Oral Radiol Endod 2005;99:743-7.

52. Tukutuku K, Muyembe-Tamfum L, Kayembe K, Mavuemba T, Sangua N, Sekele I. Prevalence of dental caries, gingivitis, and oral hygiene in hospitalized AIDS cases in Kinshasa, Zaire. J Oral Pathol Med 1990;19:271-2.

53. Schuman P, Ohmit SE, Sobel JD, Mayer KH, Geene V, Rompalo A, Klein RS. Oral lesions among women living with or at risk for HIV infection. HIV Epidemiology Research Study (HERS) Group. Am J Med 1998;104:559-64.

54. Laskaris G, Potouridou I, Laskaris M, Stratigos J. Gingival lesions of HIV infection in 178 Greek patients. Oral Surg Oral Med Oral Pathol 1992;74:168-71.

55. Rams TE, Andriolo M, Feik D, Abel SN, McGivern TM, Slots J. Microbiological study of HIV-related periodontitis. J Periodontol 1991;62:74-81.

56. Bayraktar G, Kurtulus I, Duraduryan A, Cintan S, Kazancioglu R, Yildiz A, Bural C, Bozfakioglu S, Besler M, Trablus S, Issever H. Dental and periodontal findings in hemodialysis patients. Oral Dis 2007;13:393-7.

57. Garcez J, Limeres Posse J, Carmona IT, Feijoo JF, Diz Dios P. Oral health status of patients with a mild decrease in glomerular filtration rate. Oral Surg Oral Med Oral Pathol Oral Radiol Endod 2009;107:224-8.

58. Fisher MA, Taylor GW, West BT, McCarthy ET. Bidirectional relationship between chronic kidney and periodontal disease: a study using structural equation modeling. Kidney Int 2011;79:347-55. 
59. Grossi SG, Genco RJ. Periodontal disease and diabetes mellitus: a two-way relationship. Ann Periodontol 1998;3:51-61.

60. Christgau M, Palitzsch KD, Schmalz G, Kreiner U, Frenzel S. Healing response to non-surgical periodontal therapy in patients with diabetes mellitus: clinical, microbiological, and immunologic results. J Clin Periodontol 1998;25:112-24.

61. Taylor GW. Bidirectional interrelationships between diabetes and periodontal diseases: an epidemiologic perspective. Ann Periodontol 2001;6:99-112.

62. Simpson TC, Needleman I, Wild SH, Moles DR, Mills EJ. Treatment of periodontal disease for glycaemic control in people with diabetes. Cochrane Database Syst Rev 2010:CD004714.

63. Janket SJ, Baird AE, Chuang SK, Jones JA. Meta-analysis of periodontal disease and risk of coronary heart disease and stroke. Oral Surg Oral Med Oral Pathol Oral Radiol Endod 2003;95:559-69.

64. Humphrey LL, Fu R, Buckley DI, Freeman M, Helfand M. Periodontal disease and coronary heart disease incidence: a systematic review and meta-analysis. J Gen Intern Med 2008;23:2079-86.

65. Blaizot A, Vergnes JN, Nuwwareh S, Amar J, Sixou M. Periodontal diseases and cardiovascular events: meta-analysis of observational studies. Int Dent J 2009;59:197-209.

66. Kim HD, Sim SJ, Moon JY, Hong YC, Han DH. Association between periodontitis and hemorrhagic stroke among Koreans: a case-control study. J Periodontol 2010;81:658-65.

67. Paraskevas S, Huizinga JD, Loos BG. A systematic review and meta-analyses on C-reactive protein in relation to periodontitis. J Clin Periodontol 2008;35:277-90.

68. Scannapieco FA, Papandonatos GD, Dunford RG. Associations between oral conditions and respiratory disease in a national sample survey population. Ann Periodontol 1998;3:251-6.

69. Scannapieco FA, Mylotte JM. Relationships between periodontal disease and bacterial pneumonia. J Periodontol 1996;67(10 Suppl):1114-22.

70. Scannapieco FA. Role of oral bacteria in respiratory infection. J Periodontol 1999;70:793-802.

71. Lesclous $P$, Maman L. An unusual case of a relationship between rosacea and dental foci. Oral Surg Oral Med Oral Pathol Oral Radiol Endod 1999;88:679-82.

72. Vaillant L. Critères diagnostiques de l'érysipèle. Ann Dermatol Venereol 2001;128:326-33.

73. Little JW, Rhodus NL. Dental treatment of the liver transplant patient. Oral Surg Oral Med Oral Pathol 1992;73:419-26.

74. Little JW, Falace DA, Miller CS, Rhodus NL. Dental management of the medically compromised patient, 6th ed. Mosby, St Louis, 1997.

75. Stuck AE, Minder CE, Frey FJ. Risk of infectious complications in patients taking glucocorticosteroids. Rev Infect Dis 1989;11:954-63.

76. Seppänen L, Lauhio A, Lindqvist C, Suuronen R, Rautemaa R. Analysis of systemic and local odontogenic infection complications requiring hospital care. J Infect 2008;57:116-22.

77. Bodey GP. Infection in cancer patients. A continuing association. Am J Med 1986;81:11-26.

78. Rolston KV. The Infectious Diseases Society of America 2002 guidelines for the use of antimicrobial agents in patients with cancer and neutropenia: salient features and comments. Clin Infect Dis 2004;39 Suppl 1:S44-8.

79. Lerman MA, Laudenbach J, Marty FM, Baden LR, Treister NS. Management of oral infections in cancer patients. Dent Clin North Am 2008;52:129-53.

80. Bergmann OJ, Ellegaard B, Dahl M, Ellegaard J. Gingival status during chemical plaque control with or without prior mechanical plaque removal in patients with acute myeloid leukaemia. J Clin Periodontol 1992;19:169-73.

81. Dreizen S, Menkin DJ, Keating MJ, McCredie KB, O'Neill PA. Effect of antileukemia chemotherapy on marrow, blood, and oral granulocyte counts. Oral Surg Oral Med Oral Pathol 1991;71:45-9.

82. Dreizen S, Bodey GP, Valdivieso M. Chemotherapy-associated oral infections in adults with solid tumors. Oral Surg Oral Med Oral Pathol 1983;55:113-20.

83. Raber-Durlacher JE, Epstein JB, Raber J, van Dissel JT, van Winkelhoff AJ, Guiot HF, van der Velden U. Periodontal infection in cancer patients treated with high-dose chemotherapy. Support Care Cancer 2002;10:466-73.

84. Hoen B, Alla F, Selton-Suty C, Beguinot I, Bouvet A, Briançon S, Casalta JP, Danchin N, Delahaye F, Etienne J, Le Moing V, Leport C, Minardi JL, Ruimy R, Vandenesch F; for the Association pour l'Etude et la 
Prévention de I'Endocardite infectieuse (AEPEI) Study Group. Changing profile of infective endocarditis: results of a 1-year survey in France. J Am Med Assoc 2002;288:75-81.

85. Van der Meer JT, Van Wijk W, Thompson J, Vandenbroucke JP, Valkenburg HA, Michel MF. Efficacy of antibiotic prophylaxis for prevention of native-valve endocarditis. Lancet 1992;339:135-9.

86. Strom BL, Abrutyn E, Berlin JA, Kinman JL, Feldman RS, Stolley PD, Levison ME, Korzeniowski OM, Kaye D. Risk factors for infective endocarditis: oral hygiene and nondental exposures. Circulation 2000;102:2842-8.

87. Duval X, Alla F, Hoen B, Danielou F, Larrieu S, Delahaye F, Leport C, Briançon S. Estimated risk of endocarditis in adults with predisposing cardiac conditions undergoing dental procedures with or without antibiotic prophylaxis. Clin Infect Dis 2006;42:e102-7.

88. Habib G, Hoen B, Tornos P, Thuny F, Prendergast B, Vilacosta I, Moreillon P, de Jesus Antunes M, Thilen U, Lekakis J, Lengyel M, Müller L, Naber CK, Nihoyannopouols P, Moritz A, Zamorano JL; ESC Committee for Pratice Guidelines. Guidelines on the prevention, diagnosis, and treatment of infective endocarditis (new version 2009): the Task Force on the Prevention, Diagnosis, and Treatment of Infective Endocarditis of the European Society of Cardiology (ESC). Endorsed by the European Society of Clinical Microbiology and Infectious Diseases (ESCMID) and the International Society of Chemotherapy (ISC) for Infection and Cancer. Eur Heart J 2009;30:2369-413.

89. Howell RM, Green JG. Prophylactic antibiotic coverage in dentistry: a survey of need for prosthetic joints. Gen Dent 1985;33:320-3.

90. McGowan DA, Hendrey ML. Is antibiotic prophylaxis required for dental patients with joint replacements? Br Dent J 1985;158:336-8.

91. Grant A, Hoddinott C. Joint replacement, dental surgery, and antibiotic prophylaxis. Br Med J 1992;304:959.

92. Waldman BJ, Mont MA, Hungerford DS. Total knee arthroplasty infections associated with dental procedures. Clin Orthop Relat Res 1997;343:164-72.

93. LaPorte DM, Waldman BJ, Mont MA, Hungerford DS. Infections associated with dental procedures in total hip arthroplasty. J Bone Joint Surg Br 1999;81:56-9.

94. Bauer T, Maman L, Matha C, Mamoudy P. Dental care and joint prostheses. Rev Chir Orthop Reparatrice Appar Mot 2007;93:607-18.

95. Saigal S, Doyle LW. An overview of mortality and sequelae of preterm birth from infancy to adulthood. Lancet 2008;371:261-9.

96. Moore S, Ide M, Coward PY, Randhawa M, Borkowska E, Baylis R, Wilson RF. A prospective study to investigate the relationship between periodontal disease and adverse pregnancy outcome. $\mathrm{Br}$ Dent J 2004;197:251-8; discussion 247.

97. Katz J, Chegini N, Shiverick KT, Lamont RJ. Localization of P. gingivalis in preterm delivery placenta. J Dent Res 2009;88:575-8.

98. León R, Silva N, Ovalle A, Chaparro A, Ahumada A, Gajardo M, Martinez M, Gamonal S. Detection of Porphyromonas gingivalis in the amniotic fluid in pregnant women with a diagnosis of threatened premature labor. J Periodontol 2007;78:1249-55.

99. Barak S, Oettinger-Barak O, Machtei EE, Sprecher H, Ohel G. Evidence of periopathogenic microorganisms in placentas of women with preeclampsia. J Periodontol 2007;78:670-6.

100. Rakoto-Alson S, Tenenbaum H, Davideau JL. Periodontal diseases, preterm births, and low birth weight: findings from a homogeneous cohort of women in Madagascar. J Periodontol 2010;81:205-13.

101. Nabet C, Lelong N, Colombier ML, Sixou M, Musset AM, Goffinet F, Kaminski M; Epipap Group. Maternal periodontitis and the causes of preterm birth: the case-control Epipap study. J Clin Periodontol 2010;37:37-45.

102. Ferguson JE, Hansen WF, Novak KF, Novak MJ. Should we treat periodontal disease during gestation to improve pregnancy outcomes? Clin Obstet Gynecol 2007;50:454-67.

103. Pihlstrom BL, Michalowicz BS, Johnson NW. Periodontal diseases. Lancet 2005;366:1809-20.

104. Conde-Agudelo A, Villar J, Lindheimer M. Maternal infection and risk of preeclampsia: systematic review and metaanalysis. Am J Obstet Gynecol 2008;198:7-22. 
105. Michalowicz BS, Hodges JS, DiAngelis AJ, Lupo VR, Novak MJ, Ferguson JE, Buchanan W, Bofill J, Papapanou PN, Mitchell DA, Matseoane S, Tschida PA,; OPT Study. Treatment of periodontal disease and the risk of preterm birth. N Engl J Med 2006;355:1885-94.

106. Petersson K, Söderström C, Kiani-Anaraki M, Lévy G. Evaluation of the ability of thermal and electrical tests to register pulp vitality. Endod Dent Traumatol 1999;15:127-31.

107. Ratcliff PA, Johnson PW. The relationship between oral malodor, gingivitis, and periodontitis. A review. J Periodontol 1999;70:485-9.

108. Colombier ML, Bigot C, Detienville R. Bleeding on probing. Significance - consequences. J Parodontol 1989;8:403-9.

109. Hamp SE, Nyman S, Lindhe J. Periodontal treatment of multirooted teeth. Results after 5 years. J Clin Periodontol 1975;2:126-35.

110. Bishay N, Petrikowski CG, Maxymiw WG, Lee L, Wood RE. Optimum dental radiography in bone marrow transplant patients. Oral Surg Oral Med Oral Pathol Oral Radiol Endod 1999;87:375-9.

111. HAS. Guides des indications et des procédures des examens radiologiques en odonto-stomatologie. 2006.

112. Jorge EG, Tanomaru-Filho M, Gonçalves M, Tanomaru JM. Detection of periapical lesion development by conventional radiography or computed tomography. Oral Surg Oral Med Oral Pathol Oral Radiol Endod 2008;106:e56-61.

113. Elad S, Garfunkel AA, Or R, Michaeli E, Shapira MY, Galili D. Time limitations and the challenge of providing infection-preventing dental care to hematopoietic stem-cell transplantation patients. Support Care Cancer 2003;11:674-7.

114. Wong F, Toljanic JA. A Survey of clinicians: prioritization of dental treatment in leukemia patients prior to chemotherapy. International J Prosthodont 2009;22:303-6.

115. Toljanic JA, Bedard JF, Larson RA, Fox JP. A prospective pilot study to evaluate a new dental assessment and treatment paradigm for patients scheduled to undergo intensive chemotherapy for cancer. Cancer 1999;85:1843-8.

116. Peters E, Monopoli M, Woo SB, Sonis S. Assessment of the need for treatment of postendodontic asymptomatic periapical radiolucencies in bone marrow transplant recipients. Oral Surg Oral Med Oral Pathol 1993;76:45-8.

117. Peterson DE. Pretreatment strategies for infection prevention in chemotherapy patients. $\mathrm{NCl} \mathrm{Monogr}$ 1990;9:61-71.

118. Overholser CD, Peterson DE, Williams LT, Schimpff SC. Periodontal infection in patients with acute nonlymphocyte leukemia. Prevalence of acute exacerbations. Arch Intern Med 1982;142:551-4.

119. Peterson DE, Minah GE, Overholser CD, Suzuki JB, DePaola LG, Stansburry DM, Williams LT, Schimpff SC. Microbiology of acute periodontal infection in myelosuppressed cancer patients. J Clin Oncol 1987;5:1461-8.

120. Stansbury DM, Peterson DE, Suzuki JB. Rapidly progressive acute periodontal infection in a patient with acute leukemia. J Periodontol 1988;59:544-7.

121. Guideline on dental management of pediatric patients receving chemotherapy, hematopoietic cell transplantation, and/or radiation. Pediatr Dent 2008;30(Suppl 7):219-25.

122. Borowski B, Benhamou E, Pico JL, Laplanche A, Margainaud JP, Hayat M. Prevention of oral mucositis in patients treated with high-dose chemotherapy and bone marrow transplantation: a randomised controlled trial comparing two protocols of dental care. Eur J Cancer B Oral Oncol 1994;30B:93-7.

123. Greenberg MS, Cohen SG, McKitrick JC, Cassileth PA. The oral flor as a source of septicemia in patients with acute leukemia. Oral Surg Oral Med Oral Pathol 1982;53:32-6.

124. Levy-Polack MP, Sebelli P, Polack NL. Incidence of oral complications and application of a preventive protocol in children with acute leukemia. Spec Care Dentist 1998;18:189-93.

125. Meyer U, Weingart D, Deng MC, Scheld HH, Joos U. Heart transplants - assessment of dental procedures. Clin Oral Investig 1999;3:79-83.

126. Lockhart PB, Brennan MT, Cook WH, Sasser H, Lovell RD, Skipper ER, Noll J, Cox TL, Aten DJ, Cook JW . Concomitant surgical treatment of dental and valvular heart diseases. Oral Surg Oral Med Oral Pathol Oral Radiol Endod 2009;107:71-6.

127. Mendenhall WM. Mandibular osteoradionecrosis. J Clin Oncol 2004;22:4867-8. 
128. Horiot JC, Schraub S. Systematic preservation of teeth and prophylaxis of dental decay on irradiated patients. J Radiol Electrol Med Nucl 1975;56:769-72.

129. Allard WF, el-Akkad S, Chatmas JC. Obtaining pre-radiation therapy dental clearance. J Am Dent Assoc 1993;124:88-91.

130. Epstein JB, Lunn R, Le N, Stevenson-Moore P. Periodontal attachment loss in patients after head and neck radiation therapy. Oral Surg Oral Med Oral Pathol Oral Radiol Endod 1998;86:673-7.

131. Référentiels inter-régionaux en soins oncologiques de support. Radiothérapie et soins bucco dentaires. Oncologie 2011;13:378-83.

132. Berbari EF, Osmon DR, Carr A, Hanssen AD, Baddour LM, Geene D, Kupp LI, Baughan LW, Harmsen WS, Mandrekar JN, Therneau TM, Steckelberg JM, Virk A, Wilson WR. Dental procedures as risk factors for prosthetic hip or knee infection: a hospital-based prospective case-control study. Clin Infect Dis 2010;50:8-16.

133. Danchin N, Duval X, Leport C. Prophylaxis of infective endocarditis: French recommendations 2002. Heart 2005;91:715-8.

134. Guillaud M, Blatier JF, Quillon A. Prevention of infective endocarditis: are recommendations about oral health procedures for high or moderate risk patients respected ? Rev Epidemiol Sante Publique 2005;53:398-408.

135. De Geest AF, Schoolmeesters I, Willems JL, De Geest H. An analysis of the level of dental care in cardiac patients at risk for infective endocarditis. Acta Stomatol Belg 1990;87:95-105.

136. Gutschik E, Lippert S. Dental procedures and endocarditis prophylaxis in patients with prosthetic heart valves: results of a questionnaire to 220 patients. Scand J Infect Dis 1989;21:665-8.

137. Barreira JL, Baptista MJ, Moreira J, Azevedo A, Areias JC. Understanding of endocarditis risk improves compliance with prophylaxis. Rev Port Cardiol 2002;21:939-51.

138. Duval X. Prophylaxis of infective endocarditis: trends and new recommendations. Ann Cardiol Angeiol (Paris) 2008;57:102-108.

139. Sennhenn-Kirchner S, Freund F, Grundmann S, Martin A, Borg-von Zepelin M, Christiansen H, Wolff HA, Jacobs HG. Dental therapy before and after radiotherapy - an evaluation on patients with head and neck malignancies. Clin Oral Investig 2009;13:157-64.

140. Katsura K, Sasai K, Sato K, Saito M, Hoshina H, Hayashi T. Relationship between oral health status and development of osteoradionecrosis of the mandible: a retrospective longitudinal study. Oral Surg Oral Med Oral Pathol Oral Radiol Endod 2008;105:731-8.

141. Suivi post-thérapeutique des carcinomes épidermoïdes des voies aéro-digestives supérieures de l'adulte. Recommandation pour la pratique clinique. Société française d'Oto-Rhino-Laryngologie, 2005.

142. Marx RE, Sawatari Y, Fortin M, Broumand V. Bisphosphonate-induced exposed bone (osteonecrosis/ osteopetrosis) of the jaws: risk factors, recognition, prevention, and treatment. J Oral Maxillofac Surg 2005;63:1567-75.

143. Douglas LR, Douglass JB, Sieck JO, Smith PJ. Oral management of the patient with end-stage liver disease and the liver transplant patient. Oral Surg Oral Med Oral Pathol Oral Radiol Endod 1998;86:55-64. 